\title{
Using Architecture to Reason about Information Security
}

\author{
STEPHEN CHONG, Harvard University \\ RON VAN DER MEYDEN, UNSW Australia
}

\begin{abstract}
We demonstrate, by a number of examples, that information flow security properties can be proved from abstract architectural descriptions, which describe only the causal structure of a system and local properties of trusted components. We specify these architectural descriptions of systems by generalizing intransitive noninterference policies to admit the ability to filter information passed between communicating domains. A notion of refinement of such system architectures is developed that supports top-down development of architectural specifications and proofs by abstraction of information security properties. We also show that, in a concrete setting where the causal structure is enforced by access control, a static check of the access control setting plus local verification of the trusted components is sufficient to prove that a generalized intransitive noninterference policy is satisfied.
\end{abstract}

CCS Concepts: $\bullet$ Security and privacy $\rightarrow$ Formal security models; $\bullet$ Security and privacy $\rightarrow$ Logic and verification

Additional Key Words and Phrases: Information flow security, epistemic logic, system architecture, intransitive noninterference

\section{ACM Reference Format:}

Stephen Chong and Ron van der Meyden. 2015. Using architecture to reason about information security. ACM Trans. Info. Syst. Sec. 18, 2, Article 8 (December 2015), 30 pages.

DOI: http://dx.doi.org/10.1145/2829949

\section{INTRODUCTION}

System architectures are high-level designs that describe the overall structure of a system in terms of its components and their interactions. Proposals for architectural modeling languages (e.g., AADL [2009] and Acme [Garlan et al. 2000]) vary with respect to their level of detail and contents, but at the most abstract level, architectures specify the causal structure of a system.

The Multiple Independent Levels of Security and Safety (MILS) approach [Alves-Foss et al. 2006; Vanfleet et al. 2005; Boettcher et al. 2008] proposes to use architecture as a key part of the assurance case for high-assurance systems. The details of the MILS vision are still under development [Blasum et al. 2014]. As articulated by Boettcher

An early version of this article circulated in 2009 and has been cited in the literature. The present version includes a significant reworking of results on architectural refinement from that version. A version of the paper introducing the access control implementation results was presented at the Layered Assurance Workshop at ACSAC 2012. The present version adds to these earlier versions full proofs of all results and an extended set of examples.

This work is supported by the Air Force Office of Scientific Research, by Australian Research Council Discovery grant DP1097203, and by the National Science Foundation under grant 1054172.

Authors' addresses: S. Chong, Harvard John A. Paulson School of Engineering and Applied Sciences, Harvard University, Cambridge, MA 02138; R. van der Meyden, School of Computer Science and Engineering, UNSW Australia, Sydney, NSW 2052, Australia.

Permission to make digital or hard copies of part or all of this work for personal or classroom use is granted without fee provided that copies are not made or distributed for profit or commercial advantage and that copies show this notice on the first page or initial screen of a display along with the full citation. Copyrights for components of this work owned by others than ACM must be honored. Abstracting with credit is permitted. To copy otherwise, to republish, to post on servers, to redistribute to lists, or to use any component of this work in other works requires prior specific permission and/or a fee. Permissions may be requested from Publications Dept., ACM, Inc., 2 Penn Plaza, Suite 701, New York, NY 10121-0701 USA, fax +1 (212) 869-0481, or permissions@acm.org.

(c) 2015 ACM 1094-9224/2015/12-ART8 $\$ 15.00$

DOI: http://dx.doi.org/10.1145/2829949 
et al. [2008], it encompasses a two-level design process, consisting of a policy level and a resource sharing level.

At the policy level, the system is described by an architecture in the form of a graph, in which vertices correspond to components and the edges specify permitted communication between components. In this respect, the architecture is like an intransitive noninterference security policy [Haigh and Young 1987; Rushby 1992; van der Meyden 2007]. At the policy level, one might also specify which components are trusted and the local policies that these components are trusted to enforce. According to the MILS vision, building a system according to the architecture, by composing components that satisfy their local policies, should result in the system satisfying global security and safety properties.

At the resource sharing level, MILS envisages the use of a range of infrastructural mechanisms to ensure that the architectural information flow policy is enforced despite components sharing resources such as processors, file systems, and network links. These mechanisms might include physical isolation, separation kernels, periods processing, cryptography, and separating network infrastructure. It is intended that this infrastructure will be developed to a high level of assurance so that a systems assurance case can be obtained by the composition of the assurance cases for trusted components and systems infrastructure. It is hoped this will enable a COTS-like market for infrastructural mechanisms and trusted components.

The key contribution of this article is to demonstrate, through several examples, that it is in fact possible, as envisaged in the MILS literature, to derive interesting information security properties compositionally from a high-level specification of trusted components and their architectural structure. We focus on compositional reasoning about information flow security properties.

We present a framework that allows the specification of a system architecture with local constraints on some system components. To give a precise meaning to the architectural structure, we extend the semantics for intransitive noninterference policies developed by van der Meyden [2007]. These policies are in the form of a graph, in which the vertices correspond to system components, and an edge from component $u$ to component $v$ specifies that information is permitted to flow from $u$ directly to $v$. This semantics is expressed in an asynchronous model of computation and captures a notion of information available to an attacker that does not have access to timing information. An architectural interpretation of this semantics has previously been given [van der Meyden 2012]. To express constraints on trusted components, we extend architectures by labeling edges between components with functions that further restrict the information permitted to flow along edges. One of the contributions of the work is to give a formal semantics to the enriched architectures that include these new types of edges. We also develop a theory of refinement for these enriched architectures, which enables top-down, correctness-preserving development of architectural specifications. It also enables simple proofs of information security properties on complex architectures to be obtained using an abstraction of that architecture.

We demonstrate the use of the framework through examples motivated by systems with interesting security requirements. These include multilevel secure databases, the Starlight Interactive Link [Anderson et al. 1996], a trusted downgrader, and a simple electronic election system. In each example, there is an application-specific information flow security property, expressed in a logic of knowledge, to be enforced. To enforce these properties, we identify, in each example, an architectural structure and a mathematically precise set of local constraints on the trusted components. We show for each example that its specific security property arises as a consequence of the interaction of the local constraints and the architectural structure. In each example, our results show that for any system that is compliant with the architecture, if the 
trusted components satisfy their local constraints, then the system satisfies the global information flow properties.

The information security properties presented in the examples provide informationtheoretic and application-specific guarantees. The negation of each information security property would constitute an attack in which a particular type of information flows to any attacker who is not permitted to have that information. Since the conclusions we derive are information theoretic, it follows from our results that there are no covert information channels available to an asynchronous attacker (e.g., the order of messages between components) that can violate the information security properties.

Only a few examples have been presented to date to formally justify the MILS approach to high-assurance secure systems development. One example is developed in Greve et al. [2003], but with respect to a more concrete model (based on a separation kernel formal security policy that deals with access control on memory segments) than the abstract, "noninterference"-style semantics that we consider. Our policy level model is more abstract and allows greater flexibility for implementations. However, we also consider a more concrete model, systems with structured state subject to "reference monitor conditions" [Rushby 1992]. We show that in this setting, to prove that a system complies with one of our extended architectures, it suffices to check a simple condition on the access control setting and to prove local properties of the trusted components.

By developing an abstract semantics for architectures and specifications of trusted components, and by developing additional examples, our work advances the case that global information flow security properties can be derived from a high-level systems architecture and local constraints on trusted components within this architecture, in the style of reasoning envisaged by Boettcher et al. [2008].

The structure of the article is as follows. In Section 2, we review architectures and their semantics, and extend architectures with filter functions that allow fine-grained specification of what information flows between components. This extension generalizes the semantics for intransitive noninterference of van der Meyden [2007] (Theorem 2.6). In Section 3, we introduce the epistemic logic we use to express information security properties. Additionally, we present examples of architectures and the information security properties that these architectures imply. We also discuss the difference between group knowledge and distributed knowledge, and show that information security properties defined using group knowledge are preserved under architectural refinement (Theorem 3.9) The concrete model based on access control is developed in Section 4. We extend the result of van der Meyden [2007] that showed local access control conditions enforce security (Theorem 4.1) to the extended architectures with filter functions (Theorems 4.2 and 4.3). We consider possible platforms and techniques that might be used to show the access control model holds in Section 5. We discuss related work in Section 6. Section 7 concludes. We use examples throughout to illustrate and motivate the definitions and results. Two online appendices expand on the material in the body of the article. Appendix A extends previous work on architectural refinement to account for filter functions. Appendix B gives full proofs for all results stated in the body of this work.

\section{ARCHITECTURES AND SEMANTICS}

Architectures give a policy level description of the structure of a system. We begin with a simple notion of architecture, following van der Meyden [2012]. A richer notion will be introduced later.

An architecture is a pair $\mathcal{A}=(D, \longmapsto)$, where $D$ is a set of security domains, and the binary relation $\longmapsto \subseteq D \times D$ is an information flow policy. The relation $\longmapsto$ is reflexive but not necessarily transitive. Intuitively, information is allowed to flow from domain $u$ to domain $v$ only if $u \longmapsto v$. The relation is reflexive, as it is assumed that information flow within a domain cannot be prevented, and so is always allowed. 


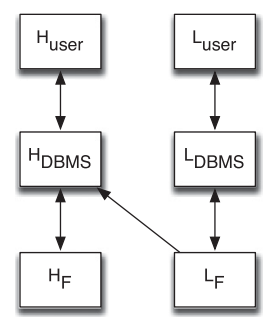

Fig. 1. Architecture $\mathcal{H S}$.

In the literature on information flow policies, domains are generally understood to correspond to security levels. We use a more general interpretation, in which domains may also correspond to system components or agents in the system. Intuitively, a domain corresponds to an interface to the system defined by the observations of the system state that may be made through that interface, and the actions that the interface allows to be performed. In an implementation of an architecture, separate domains do not necessarily utilize separate resources. Hardware, code, and data may be shared between domains. Indeed, a key challenge is ensuring that the information flow policy is respected despite the shared use of resources.

\subsection{Example: $\mathcal{H} \mathcal{L}$ Architecture}

The architecture $\mathcal{H} \mathcal{L}=(\{H, L\},\{(L, L),(H, H),(L, H)\})$ consists of two security domains $H$ and $L$, and the information flow policy indicates that information is allowed to flow from $L$ to $H$, in addition to the reflexive information flows. We can depict $\mathcal{H} \mathcal{L}$ graphically, indicating security domains with rectangles and the information flow policy with arrows. We omit arrows for reflexive information flows.

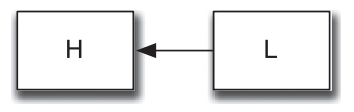

\subsection{Example: Hinke-Schaefer}

A variety of architectures have been proposed for multilevel secure database management systems (MLS/DBMS) [Thuraisingham 2005]. In the Hinke-Schaefer architecture [Hinke and Schaefer 1975], several (untrusted) single-level DBMSs are composed together in a trusted operating system. Each user interacts with a single-level DBMS. The operating system enforces access control between the single-level DBMSs, allowing more restrictive DBMSs to read the storage files of less restrictive DBMSs, but not vice versa.

Figure 1 shows architecture $\mathcal{H} \mathcal{S}$, which represents the Hinke-Schaefer architecture for two security levels at the MILS policy level. Domains $H_{\text {user }}$ and $L_{\text {user }}$ represent users of a high-security and low-security DBMS, respectively; they interact with the singlelevel DBMSs $H_{D B M S}$ and $L_{D B M S}$, respectively. The single-level DBMSs store their data in database files denoted $H_{F}$ and $L_{F}$. Note that information is allowed to flow to $H_{D B M S}$, from both $H_{F}$ and $L_{F}$, as the high-security DBMS is allowed to read the storage files of both the high-security and low-security DBMSs.

The Hinke-Schaefer architecture is also known as the "operating system providing mandatory access control" architecture [Thuraisingham 2005], as the operating system is trusted to enforce the information flows specified in the architecture. This amounts to a decision to implement the policy level architecture $\mathcal{H S}$ at the resource sharing level by means of a trusted separation kernel. 


\subsection{Machine Model}

To specify what it means for an implementation to satisfy an architecture, we must first define what an implementation is. We use the state-observed machine model [Rushby 1992], which defines deterministic state-based machines. A machine has a set of actions $A$, and each action is associated with a security domain. Intuitively, if action $a$ is associated with domain $u$, then $a$ represents a decision, choice, or action taken by the system component represented by $u$. Actions deterministically alter the state of the machine, and we assume that the observations of each security domain are determined by the current machine state.

A machine is a tuple $M=\left\langle S, s_{0}, A, D\right.$, step, obs, dom, where $S$ is a set of states, $s_{0} \in S$ is the initial state, $A$ is a set of actions, $D$ is a set of domains, step : $S \times A \rightarrow S$ is a deterministic transition function, dom : $A \rightarrow D$ associates a domain with each action, and observation function obs : $D \times S \rightarrow O$ describes for each state what observations can be made by each domain, for some set of observations $O$.

We assume that it is possible to execute any action in any state: the function step is total. Given sequence of actions $\alpha \in A^{*}$, we write $s \cdot \alpha$ for the state reached by performing each action in turn, starting in state $s$. We define $s \cdot \alpha$, inductively defined using the transition function step, by

$$
\begin{aligned}
s \cdot \epsilon & =s \\
s \cdot \alpha a & =\operatorname{step}(s \cdot \alpha, a)
\end{aligned}
$$

for $\alpha \in A^{*}$ and $a \in A$. (Here, $\epsilon$ denotes the empty sequence.) For notational convenience, we write $\mathrm{obs}_{u}$ for the function obs $(u, \cdot)$, and obs $(\alpha)$ for obs $\left(s_{0} \cdot \alpha\right)$, where $\alpha \in A^{*}$.

We use the term group to refer to a set of domains. ${ }^{1}$ Given a sequence $\alpha \in A^{*}$, the view of a group $G$ of $\alpha$ is the sequence of the group's observations and the actions that belong to members of the group. Intuitively, G's view is the history of its observations and the actions it has performed. The function view $G$ defines the view of domain $G$. We first define the observation of group $G$ at state $s$ by obs $G(s)=\left\langle\operatorname{obs}_{u}(s)\right\rangle_{u \in G}$ (i.e., the tuple of observations of individuals $u \in G$ ). The view function is then defined inductively by

$$
\begin{aligned}
\operatorname{view}_{G}(\epsilon) & =\operatorname{obs}_{G}\left(s_{0}\right) \\
\operatorname{view}_{G}(\alpha a) & = \begin{cases}\operatorname{view}_{G}(\alpha) a \operatorname{obs}_{G}(\alpha a) & \text { if } \operatorname{dom}(\alpha) \in G \\
\operatorname{view}_{G}(\alpha) \circ \operatorname{obs}_{G}(\alpha a) & \text { otherwise }\end{cases}
\end{aligned}
$$

for $\alpha \in A^{*}$ and $a \in A$. To capture that the semantics is asynchronous and insensitive to stuttering of observations, the definition uses the absorptive concatenation operator $\circ$ : for set $X$, sequence $\alpha \in X^{*}$, and element $x \in X, \alpha \circ x=\alpha$ if $x$ is equal to the last element of $\alpha$, and $\alpha \circ x=\alpha x$ otherwise. When $G=\{u\}$ is a singleton, we write view for view $_{G}$.

Finally, for any sequence of actions $\alpha \in A^{*}$, we write $\alpha \uparrow G$ for the subsequence of $\alpha$ of actions whose domain is in the set $G$.

\subsection{Semantics}

A machine satisfies an architecture if, in all possible executions of the machine, information flow is in accordance with the architecture's information flow policy. We formalize this using an approach proposed by van der Meyden [2007], which involves the use of a concrete operational model to define an upper bound on the information that a domain is permitted to learn.

The operational model is captured using a function $\mathrm{ta}_{u}$, which maps a sequence of actions $\alpha \in A^{*}$ to a representation of the maximal information that domain $u$ is permitted to have after $\alpha$, according to the policy $\longmapsto$. (Term "ta" is derived from transmission

\footnotetext{
${ }_{1}$ The term group is commonly used in the literature on the logic of knowledge to refer to a set of agents.
} 
of information about actions; the definition corrects problems identified by van der Meyden [2007] with earlier "intransitive purge"-based semantics [Rushby 1992].)

An action of $v$ should convey information to $u$ only if $v \longmapsto u$. Moreover, the information conveyed should be no more than the information that $v$ is permitted to have. Given machine $M=\left\langle S, s_{0}, A, D\right.$, step, obs, dom $\rangle$, function $\mathrm{ta}_{u}$ is defined inductively by $\operatorname{ta}_{u}(\epsilon)=$ $\epsilon$, and, for $\alpha \in A^{*}$ and $a \in A$,

$$
\operatorname{ta}_{u}(\alpha a)= \begin{cases}\operatorname{ta}_{u}(\alpha) & \text { if } \operatorname{dom}(a) \succ \not \rightarrow u \\ \left(\operatorname{ta}_{u}(\alpha), \operatorname{ta}_{\operatorname{dom}(a)}(\alpha), a\right) & \text { otherwise }\end{cases}
$$

The range of $\mathrm{ta}_{u}$ is the set of nested tuples over the basic elements $A \cup\{\epsilon\}$. Note that the result of $\operatorname{ta}_{u}(\alpha a)$ can be interpreted as a tree, where if $\operatorname{dom}(a) \longmapsto u$, the root has three children $\operatorname{ta}_{u}(\alpha)$ and $\operatorname{ta}_{\operatorname{dom}(a)}(\alpha)$ and $a$.

Note that if information is not allowed to flow from $\operatorname{dom}(a)$ to $u$, then $\operatorname{ta}_{u}(\alpha a)=\operatorname{ta}_{u}(\alpha)$ (i.e., the maximal information permitted to $u$ does not change). If information is allowed to flow from $\operatorname{dom}(a)$ to $u$, then the information conveyed is at most the information that domain $\operatorname{dom}(a)$ is permitted to have (i.e., $\operatorname{ta}_{\operatorname{dom}(a)}(\alpha)$ ), and the action $a$ that was performed. Thus, in this case, we obtain the maximal information that $u$ may have after $\alpha a$ by adding the information $\left(\operatorname{ta}_{\operatorname{dom}(a)}(\alpha), \alpha\right)$ to the maximal information $\operatorname{ta}_{u}(\alpha)$ that $u$ was permitted to have before the action $a$ was performed.

A machine is TA-compliant with an architecture if it has an appropriate set of domains, and for each domain $u$, what $u$ observes in state $s_{0} \cdot \alpha$ is determined by ta $a_{u}(\alpha)$. In other words, $\mathrm{ta}_{u}$ describes the maximal information that $u$ may learn: if in two runs $\alpha$ and $\alpha^{\prime}$ the maximal information that $u$ may learn is identical $\left(\operatorname{ta}_{u}(\alpha)=\operatorname{ta}_{u}\left(\alpha^{\prime}\right)\right)$, then $u$ 's observations in each run must be identical $\left(\mathrm{obs}_{u}(\alpha)=\mathrm{obs}_{u}\left(\alpha^{\prime}\right)\right)$.

Definition 2.1 (TA-Compliance). A system $M$ is TA-compliant with architecture $(D, \longmapsto)$ if it has domains $D$ and for all $u \in D$ and all sequences $\alpha, \alpha^{\prime} \in A^{*}$ such that $\operatorname{ta}_{u}(\alpha)=\operatorname{ta}_{u}\left(\alpha^{\prime}\right)$, we have $\operatorname{obs}_{u}(\alpha)=\operatorname{obs}_{u}\left(\alpha^{\prime}\right)$.

TA-compliance requires that if $\operatorname{ta}_{u}(\alpha)=\operatorname{ta}_{u}\left(\alpha^{\prime}\right)$, then the observations of $u$ in state $s_{0} \cdot \alpha$ and in state $s_{0} \cdot \alpha^{\prime}$ are equal. The following lemma shows that in fact TA-compliance implies that if $\operatorname{ta}_{u}(\alpha)=\operatorname{ta}_{u}\left(\alpha^{\prime}\right)$, then $\operatorname{view}_{u}(\alpha)=\operatorname{view}_{u}\left(\alpha^{\prime}\right)$.

Lemma 2.2. If $M$ is TA-compliant with respect to architecture $(D, \longmapsto)$, then for all agents $u$ and all $\alpha, \alpha^{\prime} \in A^{*}$ such that $\operatorname{ta}_{u}(\alpha)=\operatorname{ta}_{u}\left(\alpha^{\prime}\right)$, we have $\operatorname{view}_{u}(\alpha)=\operatorname{view}_{u}\left(\alpha^{\prime}\right)$.

\subsection{Filter Functions}

The architectures used so far impose coarse, global constraints on the causal structure of systems. If $u \longmapsto v$, then TA-compliance permits domain $u$ to send to domain $v$ any and all data it has. However, in many systems, key security properties depend on the fact that trusted components allow only certain information to flow from one domain to another. Finer specification of information flows in the architecture allow us to prove stronger information security properties.

We extend the notion of architecture by introducing filter functions to allow finegrained specification of what information flows between domains. We define semantics for these extended architectures and present examples where the extended architectures allow us to prove strong information security properties.

An extended architecture is a pair $\mathcal{A}=(D, \longmapsto)$, where $D$ is a set of security domains, and $\longmapsto \subseteq D \times D \times(\mathcal{L}, \cup\{\top\})$, where $\mathcal{L}$, is a set of function names. We write $u \stackrel{f}{\longmapsto} v$ when $(u, v, f) \in \longmapsto$, write $u \longmapsto v$ as shorthand for $\exists f$. $(u, v, f) \in \longmapsto$, and $u \not \succ v$ as shorthand for $\neg \exists f .(u, v, f) \in \longmapsto$. 
Intuitively, $u \stackrel{f}{\longmapsto} v$ represents that information flow from $u$ to $v$ is permitted but may be subject to constraints. In case $f=T$, there are no constraints on information flow from $u$ to $v$ : any information that may be possessed by $u$ is permitted to be passed to $v$ when $u$ acts, just as in the definition of TA-compliance. If $f \in \mathcal{L}$, then information is allowed to flow from domain $u$ to domain $v$, but it needs to be filtered through the function denoted by $f$ : only information output by this function may be transmitted from $u$ to $v$. If $u \not t v v$, then no direct flow of information from $u$ to $v$ is permitted.

In some cases, it may be possible for the operating system or network infrastructure to enforce a given filter function. However, in general, a filter function is a local constraint on a trusted component of the system. In other words, if $u \stackrel{f}{\longmapsto} v$ for $f \neq \top$, then component $u$ is trusted to enforce that information sent to $v$ is filtered appropriately.

We require that extended architectures have the following properties:

(1) For all $u, v \in D$, there exists at most one $f \in \mathcal{L}, \cup\{\top\}$ such that $u \stackrel{f}{\longmapsto} v$.

(2) The relation $\longmapsto$ is reflexive in that for all $u \in D$ we have $(u, u, \top) \in \longmapsto$.

The first condition requires that all permitted flows of information from $u$ to $v$ are represented using a single labeled edge. Intuitively, any policy with multiple such edges can always be transformed into one satisfying this condition by combining the pieces of information flowing across these edges into a tuple that flows across a single edge. The second condition is motivated from the fact, already noted earlier, that information flow from a domain to itself cannot be prevented.

For example, the following diagram shows an extended architecture with domains $H, D$, and $L$, intended to represent a high-security domain, a trusted declassifier, and a low-security domain, respectively. The arrows indicate permitted flow between domains. The label on the edge from $D$ to $L$ indicates that information going from $D$ to $L$ should be filtered by function rel. When drawing extended architectures, we annotate arrows between domains with the filter function names. For arrows drawn without a label, and elided reflexive arrows, the implied label is $T$. This allows unextended architecture diagrams to be interpreted as extended architecture diagrams. (Note that this diagrammatic convention differs from our convention in textual contexts where, for extended policies $\longmapsto$, we write $u \longmapsto v$ if there exists $f \in \mathcal{L} \cup\{\top\}$ such that $(u, v, f) \in \longmapsto$.)

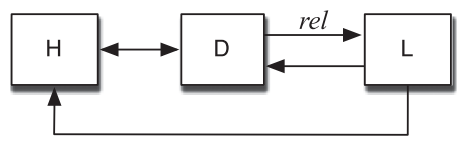

Extended architectures do not define the interpretations of the function names $\mathcal{L}$. If $\mathcal{A}=(D, \longmapsto)$ is an extended architecture, an interpretation for $\mathcal{A}$ is a tuple $\mathcal{I}=(A$, dom, $I)$, where $A$ is a set of actions, dom : $A \rightarrow D$ assigns these actions to domains of the architecture $\mathcal{A}$, and $\mathrm{I}$ is a function mapping each $f \in \mathcal{L}$, to a function with domain $A^{*} \times A$ (and arbitrary codomain). In other words, for each $f \in \mathcal{L}$, the function $\mathrm{I}(f)$ is a function that maps a sequence of actions and an action to some value. We call the pair $(\mathcal{A}, \mathcal{I})$ an interpreted extended architecture, or simply an interpreted architecture.

Intuitively, if $u \stackrel{f}{\longmapsto} v$ and $\alpha \in A^{*}$ and $a \in A$ is an action with $\operatorname{dom}(\alpha)=u$, then $\mathrm{I}(f)(\alpha, a)$ is the information that is permitted to flow from $u$ to $v$ when the action $a$ is performed after occurrence of the sequence of actions $\alpha \in A^{*}$. We allow the functions $\mathrm{I}(f)$ to have arbitrary codomains to allow flexibility in modeling the concrete data values transmitted by various applications. However, for the purposes of reasoning about information security properties, if function $h$ is the interpretation of a filter function, 
the important information about $h$ is how its inverse $h^{-1}$ partitions domain $A^{*} \times A$. In other words, if $h(\alpha, a)=h(\beta, b)$ (for sequences $\alpha, \beta \in A^{*}$ and actions $a, b \in A$ ), then $(\alpha, a)$ and $(\beta, b)$ are in the same partition imposed by $h^{-1}$, and thus $h$ reveals the same information about trace $\alpha a$ as it does about trace $\beta b$. There is one special case where the value of $\mathrm{I}(f)(\alpha, a)$ does carry significance, which is when it takes the special value $\epsilon$ (the empty sequence). This case corresponds to the transmission of no information (not even the fact that an action has been performed), and we use the null string because it has the effect, in the following definitions, of leaving unchanged a prior state of information represented as a string.

Given extended architecture $(D, \longmapsto)$ and an architectural interpretation $\mathcal{I}=$ ( $A$, dom, I), we define a function $\mathrm{fta}_{u}$ with domain $A^{*}$ that, like $\mathrm{ta}_{u}$, captures the maximal information that domain $u$ is permitted to have after a sequence of actions has been executed. The definition is recursive with a function $\mathrm{T}_{v, u}$ for $u, v \in D$, mapping a sequence $\alpha \in A^{*}$ and an action $a \in A$ with $\operatorname{dom}(a)=v$ to

$$
\mathrm{T}_{v, u}(\alpha, a)= \begin{cases}\epsilon & \text { if } v \not \nrightarrow u \\ \left(\mathrm{fta}_{v}(\alpha), a\right) & \text { if } v_{\succ}^{\top} u \\ \mathrm{I}(f)(\alpha, a) & \text { if } v_{\succ}{ }_{\hookrightarrow} u .\end{cases}
$$

Intuitively, $\mathrm{T}_{v, u}(\alpha, a)$ represents the new information permitted to be known by $u$ when action $\alpha$ is performed after sequence $\alpha$. As discussed earlier, the value $\mathrm{I}(f)(\alpha, a)$ is a concrete representation of the information that may be transmitted from $v$ to $u$ across an $f$-labeled edge when action $a$ is performed after sequence $\alpha$. This term takes the specific value $\epsilon$ when no information may be transmitted. Since the absence of an edge from $v$ to $u$ implies that there is always a prohibition of direct information flow from $v$ to $u$, in this case $\mathrm{T}_{v, u}(\alpha, a)$ always takes the value $\epsilon$. In the case of a T-labeled edge, there are no constraints on the flow of information from $v$ to $u$. Here, the definition models that $v$ transmits all of its permitted information (represented by $\mathrm{fta}_{v}(\alpha)$ ) as well as the fact that the action $a$ is being performed.

The function $\mathrm{fta}_{u}$ is defined by $\mathrm{fta}_{u}(\epsilon)=\epsilon$, and, for $\alpha \in A^{*}$ and $a \in A$, $\operatorname{fta}_{u}(\alpha a)=$ $\mathrm{fta}_{u}(\alpha)^{\wedge} \mathrm{T}_{\operatorname{dom}(a), u}(\alpha, \alpha)$ where ${ }^{\wedge}$ is the operation of appending an element to the end of a sequence. Some important technical points concerning the append operation are that for any sequence $\sigma$, we define $\sigma^{\wedge} \epsilon=\sigma$ (i.e., appending the empty sequence $\epsilon$ has no effect), and if $\delta$ happens to be a nonempty sequence, then $\sigma^{\wedge} \delta$ is the sequence that extends the sequence $\sigma$ by the single additional element $\delta$. For example, if $\delta$ is the sequence $a b$, then $\sigma^{\wedge} \delta$ has final element equal to the sequence $a b$ rather than $b$.

Unfolding the definition, we obtain

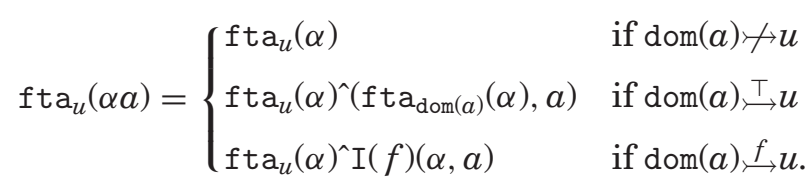

The first two clauses resemble the definition of $\mathrm{ta}_{u}$; the third adds to this that the information flowing along an edge labeled by a function name $f$ is filtered by the interpretation $\mathrm{I}(f)$. Note that if $\mathrm{I}(f)(\alpha, a)=\epsilon$, where $\operatorname{dom}(a) \stackrel{f}{\rightarrow} u$, then $\mathrm{fta}_{u}(\alpha a)=\mathrm{fta}_{u}(\alpha)$. In other words, filter function $\mathrm{I}(f)$ can specify that no information should flow under certain conditions. Note also that $\mathrm{fta}_{u}$ and $\mathrm{T}_{v, u}$ have implicit parameters, namely an information flow policy $\longmapsto$ and an architectural interpretation $\mathcal{I}=(A$, dom, I). When we need to make some of these parameters explicit, we write expressions such as $f \mathrm{ta}(\hookrightarrow, \mathcal{I})$ or $\operatorname{fta}_{u}^{\longleftrightarrow}$. 
The function $\mathrm{fta}_{u}$ is used analogously to $\mathrm{ta}_{u}$ to define the maximal information that a domain is permitted to observe for a given sequence of actions. However, $\mathrm{fta}_{u}$ is a more precise bound than $\mathrm{ta}_{u}$, as it uses filter functions to bound the information sent between domains.

It is reasonable to assume that information sent from $u$ to $v$ is information that $u$ is permitted to have. We say that a function is $\mathrm{fta}_{u}$-compatible when the information it conveys is determined by information that $u$ is permitted to have.

Definition 2.3. Function $h$ with domain $A^{*} \times A$ is $\mathrm{fta}_{u}$-compatible when for all sequences $\alpha, \beta \in A^{*}, \mathrm{fta}_{u}(\alpha)=\mathrm{fta}_{u}(\beta) \operatorname{implies}$ that for all $a \in A$ with $\operatorname{dom}(a)=u$, we have $h(\alpha, a)=h(\beta, a)$.

If a function $h$ is not $\mathrm{fta}_{u}$-compatible, then it might reveal information that domain $u$ is not permitted to know or might not even possess. For example, if there are traces $\alpha, \beta \in A^{*}$ such that domain $u$ is permitted to have the same information in both traces (i.e., $\left.\mathrm{fta}_{u}(\alpha)=\mathrm{fta}_{u}(\beta)\right)$, but $h(\alpha, a) \neq h(\beta, a)$, then it is possible that the value of $h(\alpha, a)$ is determined by actions that occurred in some other domain $v$ that domain $u$ knows nothing about. Indeed, there may be no directed path in the architecture from $v$ to $u$, implying that $u$ never learns anything about $v$. The concept of $\mathrm{fta}_{u}$-compatibility allows us to rule out such functions that cannot be realized in any implementation of the architecture.

We say that the interpretation $\mathcal{I}=(A$, dom, I $)$ is compatible with $\mathcal{A}=(D, \longmapsto)$ if for all

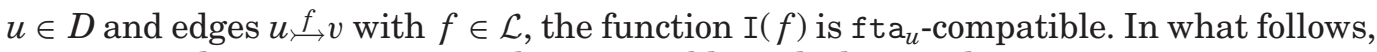
we require that interpretations be compatible with their architectures.

A machine complies with an interpreted extended architecture if it has appropriate domains and actions, and for each domain $u$, what $u$ observes in state $s_{0} \cdot \alpha$ is determined by $\mathrm{fta}_{u}(\alpha)$. We call such a machine FTA-compliant. ("FTA" is derived from filtered transmission of information about actions.)

Definition 2.4 (FTA-Compliant). A machine $M=\left\langle S, s_{0}, A, D\right.$, step, obs, dom $\rangle$ is FTA-compliant with an interpreted architecture $(\mathcal{A}, \mathcal{I})$, with $\mathcal{A}=\left(D^{\prime}, \longmapsto\right)$ and $\mathcal{I}=\left(A^{\prime}\right.$, dom $^{\prime}$, I), if $A=A^{\prime}, D=D^{\prime}$, dom $=$ dom $^{\prime}$ and for all agents $u \in D$ and all $\alpha, \alpha^{\prime} \in A^{*}$, if $\operatorname{fta}_{u}(\alpha)=\mathrm{fta}_{u}\left(\alpha^{\prime}\right)$, then $\operatorname{obs}_{u}(\alpha)=\operatorname{obs}_{u}\left(\alpha^{\prime}\right)$.

Separating extended architectures from their interpretations ensures that extended architectures can be completely represented by graphical diagrams with labeled edges. It also allows us to deal with examples where an extended architecture can be implemented in a variety of ways, and weak constraints on the set of actions and the set of filter functions suffice to enforce the security properties of interest. We will present several examples of this in what follows. To capture the constraints on the architectural interpretations at the semantic level, we use the notion of an architectural specification, which is a pair $(\mathcal{A}, \mathcal{C})$ where $\mathcal{A}$ is an extended architecture and $\mathcal{C}$ is a set of architectural interpretations for $\mathcal{A}$. (In this work, we will not attempt to develop any syntactic notation for architectural specifications.)

Definition 2.5. A machine $M$ is FTA-compliant with an architectural specification $(\mathcal{A}, \mathcal{C})$ if there exists an interpretation $\mathcal{I} \in \mathcal{C}$ such that $M$ is FTA-compliant with the interpreted architecture $(\mathcal{A}, \mathcal{I})$.

The following theorem shows that FTA-compliance generalizes TA-compliance. Thus, we are free to interpret a given architecture as an extended architecture.

THEOREM 2.6. Let $\mathcal{A}_{1}=\left(D, \longmapsto_{1}\right)$ be an architecture, and let $\mathcal{A}_{2}=\left(D, \longmapsto_{2}\right)$ be the extended architecture such that $(u, v, f) \in \longmapsto_{2}$ if and only if $f=\top$ and $(u, v) \in \longmapsto_{1}$. Let $M$ be a machine with domains $D$, actions $A$, and domain function dom. Let $\mathcal{I}=(A$, dom, $I)$ 
be any interpretation for $\mathcal{A}_{2}$ with this set of actions and domain function. Then $M$ is TA-compliant with $\mathcal{A}_{1}$ if and only if $M$ is FTA-compliant with $\left(\mathcal{A}_{2}, \mathcal{I}\right)$.

FTA-compliance requires that if $\mathrm{fta}_{u}(\alpha)=\mathrm{fta}_{u}\left(\alpha^{\prime}\right)$, then the observations of $u$ in state $s_{0} \cdot \alpha$ and in state $s_{0} \cdot \alpha^{\prime}$ are equal. The following lemma (similar to Lemma 2.2) shows that in fact FTA-compliance implies that if $\operatorname{fta}_{u}(\alpha)=\mathrm{fta}_{u}\left(\alpha^{\prime}\right)$, then we have that $\operatorname{view}_{u}(\alpha)=\operatorname{view}_{u}\left(\alpha^{\prime}\right)$.

We require a technical assumption for this result.

Definition 2.7. An interpreted architecture $(\mathcal{A}, \mathcal{I})$ is nonconflating if for all $u, v \in D$, if $u \stackrel{f}{\rightarrow} v$ with $f \neq \top$, then for all actions $a, b \in A$ with $\operatorname{dom}(a)=u$ and $\operatorname{dom}(b)=v$, and for all $\alpha, \beta \in A^{*}$ we have $\mathrm{I}(f)(\alpha, a) \neq\left(\mathrm{fta}_{v}(\beta), b\right)$.

Recall that by reflexivity, $u \stackrel{f}{\rightarrow} v$ with $f \neq \top$ implies that $u \neq v$. Intuitively, the condition states that in the context of the definition of $\mathrm{fta}_{v}$, it is always possible for domain $v$ to distinguish the type of information $\mathrm{I}(f)(\alpha, \alpha)$ transmitted to it from the type of information $\left(\mathrm{fta}_{v}(\beta), b\right)$ transmitted by $v$ to itself. In other words, $v$ can distinguish between the effects of its own actions on $\mathrm{fta}_{v}$ and the effects of other domains' actions. Since, intuitively, $v$ should be aware of its own actions, it is reasonable to expect that this is generally satisfied.

LEMMA 2.8. If $M$ is FTA-compliant with nonconflating interpreted architecture $(\mathcal{A}, \mathcal{I})$, with $\mathcal{A}=(D, \longmapsto)$ and $\mathcal{I}=(A$, dom, I $)$, then for all agents $u \in D$ and all $\alpha, \alpha^{\prime} \in A^{*}$ such that $\operatorname{fta}_{u}(\alpha)=\operatorname{fta}_{u}\left(\alpha^{\prime}\right)$, we have $\operatorname{view}_{u}(\alpha)=\operatorname{view}_{u}\left(\alpha^{\prime}\right)$.

We note that this result does not hold for conflating interpreted architectures. For example, consider an interpreted architecture in which $\operatorname{dom}(a) \stackrel{f}{\rightarrow} \operatorname{dom}(b)$ and $\mathrm{I}(f)(\alpha, a)=$ $(\epsilon, b)$ and a system in which obs $(s)=\perp$ for all $u \in D$ and states $s$. This system is necessarily FTA-compliant with the architecture, but we have $\operatorname{fta}_{\operatorname{dom}(b)}(a)=(\epsilon, b)=$ $\mathrm{fta}_{\mathrm{dom}(b)}(b)$ but view $\operatorname{dom}(b)(a)=\perp$ and $\operatorname{view}_{\operatorname{dom}(b)}(b)=\perp b \perp$.

However, it is always possible to convert an interpretation I into another equivalent nonconflating interpretation $\mathrm{I}^{\prime}$ by defining $\mathrm{I}^{\prime}(f)(\alpha, a)=(\mathrm{I}(f)(\alpha, a), x)$ for some fixed value $x$ that is not in $A$. Note that I and I' are equivalent in the sense that $\mathrm{I}(f)(\alpha, a)=$ $\mathrm{I}(g)(\beta, b)$ if and only if $\mathrm{I}^{\prime}(f)(\alpha, \alpha)=\mathrm{I}^{\prime}(g)(\beta, b)$, so the "information content" of the values of I and I' are the same. We therefore assume in what follows that interpreted architectures are nonconflating.

\section{INFORMATION SECURITY PROPERTIES}

We use a (fairly standard) propositional epistemic logic [Fagin et al. 1995] to express information security properties. In general, a richer logic (e.g., incorporating also temporal operators) is likely to be useful. We confine ourselves to a level of expressiveness that suffices for the applications we present.

The syntax is defined as follows:

$$
\begin{aligned}
\phi, \psi & :=\top|p| \neg \phi|\phi \wedge \psi| K_{G} \phi \\
& G \text { ranges over groups of domains. }
\end{aligned}
$$

In case $G=\{u\}$ is a singleton, we write simply $K_{u} \phi$ for $K_{G} \phi$.

Formulas $\top, p, \neg \phi$, and $\phi \wedge \psi$ are standard from propositional logic: $\top$ is always satisfied, and $p$ is a propositional constant from a set $\Phi$. Epistemic formula $K_{G} \phi$ says that the group of domains $G$, considered as a single domain, knows $\phi$. We write $\mathcal{L}(\Phi)$ for the set of formulas described earlier (i.e., the formulas that can be built up starting from $T$ and the primitive propositions in $\Phi$ ) using conjunction, negation, and modal operators $K_{G}$. 


$$
\begin{aligned}
& M, \pi, \alpha \vDash \top \\
& M, \pi, \alpha \vDash p \quad \text { if and only if } \alpha \in \pi(p) \\
& M, \pi, \alpha \vDash \neg \phi \quad \text { if and only if } M, \pi, \alpha \not \models \phi \\
& M, \pi, \alpha \vDash \phi \wedge \psi \text { if and only if } M, \pi, \alpha \vDash \phi \text { and } M, \pi, \alpha \vDash \psi \\
& M, \pi, \alpha \vDash K_{G} \phi \quad \text { if and only if } M, \pi, \alpha^{\prime} \vDash \phi \text { for all } \alpha^{\prime} \in A^{*} \text { s.t. } \alpha \approx_{G} \alpha^{\prime}
\end{aligned}
$$

Fig. 2. Epistemic logic semantics.

Formulas are interpreted using a possible worlds semantics, where a world is a sequence of actions $\alpha \in A^{*}$. A proposition is a set $X \subseteq A^{*}$. We say that proposition $X$ is nontrivial if $X \neq \varnothing$ and $X \neq A^{*}$. An interpretation function $\pi$ is a function from propositional constants to propositions.

We define the semantics of the logic using satisfaction relation $M, \pi, \alpha \vDash \phi$, which intuitively means that formula $\phi$ is true given interpretation function $\pi$, and machine $M$ that has executed sequence $\alpha \in A^{*}$. Figure 2 defines relation $M, \pi, \alpha \vDash \phi$. To interpret epistemic formulas $K_{G} \phi$, we use an indistinguishability relation for each group of domains $G$, which describes what sequences of actions $G$ considers possible given its view of the actual sequence of actions. Two sequences of actions $\alpha \in A^{*}$ and $\alpha^{\prime} \in A^{*}$ are indistinguishable to group of domains $G$, written $\alpha \approx_{G} \alpha^{\prime}$, if $G$ 's views of the two sequences are identical: $\alpha \approx_{G} \alpha^{\prime} \Longleftrightarrow \operatorname{view}_{G}(\alpha)=\operatorname{view}_{G}\left(\alpha^{\prime}\right)$. Note that the machine $M$ determines both this relation and the allowable sequences $\alpha$. Note also that when $G$ is not a singleton, then the knowledge that group $G$ has is based not only on the events that members of $G$ observe but also on the total ordering of these events. We remark that the operator $K_{G}$ differs from the usual "distributed knowledge" operator, which uses only a partial ordering: we explain this later in Section 3.6.

We write $M, \pi \vDash \phi$ if for all $\alpha \in A^{*}$ we have $M, \pi, \alpha \vDash \phi$. We say that $\phi$ is valid if $M, \pi \vDash \phi$ for all systems $M$ and interpretations $\pi$.

$G$-dependent propositions. A proposition in a system $M$ depends on the actions of a group $G$ if its truth value can be affected by making changes only to the actions of domains in the group $G$. The notion of dependence of a proposition on a group $G$ is useful to specify confidential information in some of our examples.

Formally, for a group $G$ of domains and $\alpha \in A^{*}$, say that a proposition $X \subseteq A^{*}$ depends on $G$ actions at $\alpha$ if there exists $\beta \in A^{*}$ such that $\alpha \uparrow \bar{G}=\beta \uparrow \bar{G}$ but $\alpha \in X$ if and only if $\beta \notin X$. (Notation $\bar{G}$ is shorthand for the set $D \backslash G$ and denotes the set of all domains excluding those in $G$.) Intuitively, this says that which $G$ actions have occurred, and their placement with respect to the actions of other domains, can affect whether or not the proposition holds. We say that $X$ depends everywhere on $G$ actions if $X$ depends on $G$ actions at $\alpha$ for all $\alpha \in A^{*} .^{2}$

The logic allows us to state information security properties about machines in terms of the knowledge of domains. We now demonstrate by several examples that an architecture can provide sufficient structure to prove that a given information security property holds in all machines that comply with the architecture. We begin with two simple examples that show that this is already the case for the simpler notion of architecture interpreted using TA-compliance before proceeding to three more elaborate examples where extended architectures and FTA-compliance are required.

\footnotetext{
${ }^{2}$ In earlier versions of this work, we used nontrivial $G$-action local propositions. A proposition $X$ is $G$-action local if for all $\alpha, \beta \in A^{*}$, if $\alpha \uparrow G=\beta\lceil G$, then $\alpha \in X \Longleftrightarrow \beta \in X$. It can easily be seen that a nontrivial $G$-action local proposition is everywhere dependent on $G$ actions. Consequently, a formulation of our results using propositions that depend on $G$ actions is more general.
} 


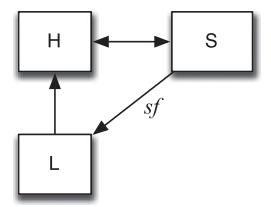

Fig. 3. Architecture $\mathcal{S L}$.

\subsection{Example: $\mathcal{H} \mathcal{L}$ Information Security}

Using the $\mathcal{H} \mathcal{L}$ architecture, we are able to show that in any execution of any machine that complies with $\mathcal{H} \mathcal{L}$, the domain $L$ does not know any proposition that depends on $H$ actions.

Proposition 3.1. If $M$ is TA-compliant with $\mathcal{H} \mathcal{L}$ and $\pi(p)$ depends on $H$ actions at $\alpha$ then $M, \pi, \alpha \vDash \neg K_{L} p$.

Note that although this result is stated using only propositional constants $p$, it in fact follows for any formula $\phi$ that if $M$ is TA-compliant with $\mathcal{H} \mathcal{L}$ and the meaning $X(\phi, M, \pi)=\left\{\beta \in A^{*} \mid M, \pi, \beta \vDash \phi\right\}$ of $\phi$ in $M$ with respect to $\pi$ depends on $H$ actions at $\alpha$, then $M, \pi, \alpha \vDash \neg K_{L} \phi$. This is because the quantification over propositions is stated at the semantic level, and we may take $\pi(p)=X(\phi, M, \pi)$ for a fresh propositional constant $p$. Our statement using propositional constants is therefore as general as would be a statement that quantified over formulas and interpretations. We frequently use results in this pattern in the sequel.

\subsection{Example: Hinke-Schaefer}

In the Hinke-Schaefer database architecture $\mathcal{H} \mathcal{S}$, none of the domains $L_{u s e r}, L_{D B M S}$, or $L_{F}$ know anything about the domains $H_{u s e r}, H_{D B M S}$, or $H_{F}$. This is true even if we consider the group knowledge of $L_{u s e r}, L_{D B M S}$, and $L_{F}$.

Proposition 3.2. Let system $M$ be TA-compliant with $\mathcal{H} \mathcal{S}$, and let $G=$ $\left\{L_{\text {user }}, L_{D B M S}, L_{F}\right\}$. If $\pi(p)$ depends on $\left\{H_{u s e r}, H_{D B M S}, H_{F}\right\}$ actions at $\alpha$, then $M, \pi, \alpha \vDash$ $\neg K_{G} p$.

Since $K_{u} p \Rightarrow K_{G} p$ is valid for $u \in G$, it follows that also $M, \pi \vDash \neg K_{u} p$ for $u \in$ $\left\{L_{\text {user }}, L_{D B M S}, L_{F}\right\}$. In particular, $L_{\text {user }}$ does not have any information about the high side of the system.

\subsection{Example: Starlight Interactive Link}

The Starlight Interactive Link [Anderson et al. 1996] provides interactive access from a high-security network to a low-security network. This allows a user on the highsecurity network to have windows open on her screen at differing security levels while ensuring that no high-security information goes to the low-security network.

Starlight has both hardware and software components. The hardware device is connected to both the high-security and low-security networks, and has a keyboard and mouse attached. There is a switch that can toggle between the high-security and lowsecurity networks; input from the mouse and keyboard are sent to the network currently selected by the switch. Starlight allows data from the low-security network to be transferred to the high-security network, but not vice versa. The Starlight software components include proxy window clients and servers to allow the windowing environment to work in the presence of the Starlight hardware.

Figure 3 shows extended architecture $\mathcal{S L}$, an architecture for the Starlight Interactive Link. The architecture uses a filter function to specify what information the Starlight Interactive Link may send to the low-security network. 
Domain $H$ represents the high-security network (including the user's computer); domain $L$ represents the low-security network; domain $S$ represents the Starlight Interactive Link (including input devices), which routes keyboard and mouse events to either the high-security or low-security network. Note that there is no edge from domain $L$ to domain $S$, as no information is sent directly from the low-security network to the Starlight Interactive Link. Instead, data from the low-security network (e.g., updates to the contents of a window) are sent to the high-security network and therefore to software components of the Starlight Interactive Link.

The edge labeled $s f$ restricts what information is allowed to flow from the Starlight Interactive Link to low-security network $L$. We present an architectural specification $\mathcal{C}_{\mathcal{S L}}$ based on $\mathcal{S L}$ that expresses a constraint on interpretations of $s f$. An interpretation $\left(A\right.$, dom, I) is included in $\mathcal{C}_{\mathcal{S L}}$ if the following conditions hold. Let $A_{S}=\{a \in A \mid \operatorname{dom}(a)=$ $S$ \} be the set of actions belonging to domain $S$. We assume that there is a distinguished action $t \in A_{S}$ that toggles which network is receiving the input events. Intuitively, $L$ is permitted to know about the occurrence of any $t$ action and the occurrence of any other action in $A_{S}$ (e.g., keyboard or mouse input) that happens while the low-security network is selected (i.e., after an odd number of toggle actions). We capture this by the following assumption on interpretation I:

$$
\mathrm{I}(s f)(\alpha, a)= \begin{cases}a & \text { if } a=\mathrm{t} \text { or } \\ & \#_{\mathrm{t}}(\alpha) \text { is odd and } a \in A_{S} \\ \epsilon & \text { otherwise, }\end{cases}
$$

where $\#_{\mathrm{t}}(\alpha)$ is the number of occurrences of $t$ in $\alpha$. For example, with the $\alpha_{i}$ consisting of only $H$ and $L$ actions, and $a \neq t$ an $S$ action, we have

$$
\begin{aligned}
& \mathrm{I}(s f)\left(\alpha_{0}, a\right)=\epsilon, \\
& \mathrm{I}(s f)\left(\alpha_{0} a, \mathrm{t}\right)=t, \\
& \mathrm{I}(s f)\left(\alpha_{0} a \mathrm{t} \alpha_{1}, a\right)=a, \text { and } \\
& \mathrm{I}(s f)\left(\alpha_{0} a \mathrm{t} \alpha_{1} a \mathrm{t} \alpha_{2}, a\right)=\epsilon, \text { and so on. }
\end{aligned}
$$

It is straightforward to check for such an interpretation that it is nonconflating and that $I(s f)$ is $\mathrm{fta}_{S}$-compatible.

The component $S$, corresponding to the Starlight Interactive Link, is a trusted component, and the $s f$ filter is a local constraint on the component. To verify that a system satisfies specification $\left(\mathcal{S} \mathcal{L}, \mathcal{C}_{\mathcal{S L}}\right)$, we would need to verify that $S$ appropriately filters information sent to $L$, and that all other communication in the system complies with the architecture, to wit, that $H$ cannot communicate directly with $L$, and $L$ cannot communicate directly with $S$.

If a system does satisfy specification $\left(\mathcal{S} \mathcal{L}, \mathcal{C}_{\mathcal{S L}}\right)$, then we can show that domain $L$ never knows any $H$-dependent propositions. Indeed, we can show something stronger, that $L$ never knows any proposition about $H$ actions and $S$ actions that occur while the high network is selected.

To fully capture this intuition requires a little care, since a proposition such as " $H$ did action $a$ between the first and second t actions" should not be known to $L$, but refers both to something that should be hidden from $L$ and to something that $L$ is permitted to observe (the $t$ actions). We handle this using an approach that is similar to the way we used $G$-dependent propositions earlier.

Let the canonical form of a sequence $\alpha \in A^{*}$ be the (unique) representation $\alpha=$ $\alpha_{0} t \alpha_{1} t \alpha_{2} \ldots t \alpha_{n}$ where each $\alpha_{i}$ contains no t actions. Define $\operatorname{tog} L: A^{*} \rightarrow A^{*}$ to be the function such that if $\alpha=\alpha_{0} t \alpha_{1} t \alpha_{2} \ldots t \alpha_{n}$ is the canonical form, then

$$
\operatorname{tog} L(\alpha)=\left(\alpha _ { 0 } \lceil L ) \mathrm { t } ( \alpha _ { 1 } \uparrow L S ) \mathrm { t } \left(\alpha_{2}\lceil L) \mathrm{t} \ldots \mathrm{t}\left(\alpha_{n} \uparrow G_{n}\right),\right.\right.
$$


where $G_{n}=L$ if $n$ is even and $G_{n}=L S$ if $n$ is odd. Intuitively, this is the subsequence consisting of the events that $L$ is permitted to observe. All other events in $\alpha$ are $H$ events, and $S$ events that occurred while the system was toggled to high. We say that a proposition $X$ is toggle-high dependent at $\alpha$ if there exists a sequence $\beta \in A^{*}$ such that $\operatorname{tog} L(\alpha)=\operatorname{tog} L(\beta)$ but $\alpha \in X$ if and only if $\beta \notin X$. Intuitively, this says that changing $\alpha$ by adding or deleting $H$ events and $S$ events that $L$ is not permitted to know about, we can change the value of the proposition $X^{3}$

Proposition 3.3. Suppose that system M is FTA-compliant with architectural specification $\left(\mathcal{S} \mathcal{L}, \mathcal{C}_{\mathcal{S L}}\right)$. If $\pi(p)$ is a toggle-H dependent proposition at $\alpha$, then $M, \pi, \alpha \vDash \neg K_{L} p$.

Note that we would not be able show this security property in an architecture without filter functions, since the domain $S$ can communicate with both $H$ and $L$. It is the filter function that allows us to show that $S$ 's communication with $L$ reveals nothing about $H$ actions and only limited information about $S$ actions.

\subsection{Example: Downgrader}

Many systems downgrade information, releasing confidential information to untrusted entities. Because it is a sensitive operation, downgrading is typically restricted to certain trusted components, called downgraders.

The following diagram shows extended architecture $\mathcal{D G}$, containing low-security domain $L$, high-security data store $H$ for two domains of high-security users $C$ and $P$, and downgrader $D$.

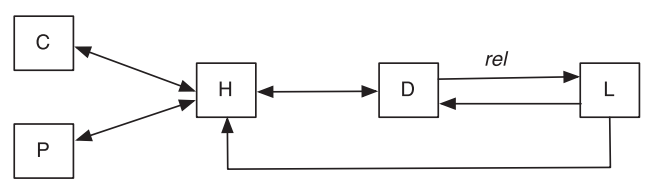

This architecture represents a system where some information is allowed to flow from the high-security domain $H$ to low-security domain $L$, but only through the downgrader $D$. In particular, we assume that domain $L$ is permitted to know about the actions of $P$ (via downgrader $D$ ) but should never know anything about the actions of $C$. This may be an appropriate model for a government agency where some sensitive information may be released to the public, but certain sensitive data (e.g., the identity and activity of covert employees, represented by domain $C$ ) should never be released.

The filter function for rel restricts the information that may be released from $D$ to $L$. It is a local constraint on the trusted component $D$. The filter function should ensure that nothing is revealed about the actions of $C$. We define an architectural specification $\mathcal{C}_{\mathcal{D G}}$ to restrict our attention to architectural interpretations with suitable filter functions.

We first define an interpretation of the function rel stating that the information transmitted across this edge is the maximum information that $D$ would have if the domain $C$ were completely cut off from the other domains. Let $\iota^{\prime}$ be the information flow policy such that $(u, v, f) \in \succ^{\prime}$ if and only if $f=\top$ and either $u=v=C$ or there

\footnotetext{
${ }^{3}$ This definition of toggle-high dependent differs from that used in the earlier version of this work published at LAW '12. The present formulation simplifies and strengthens the corresponding result, Proposition 3.3.
} 
exists an edge $(u, v, g) \in \longmapsto$ and $\{u, v\} \subseteq\{P, H, D, L\}$. This is the policy depicted in the following diagram, where, as usual, we omit reflexive edges:

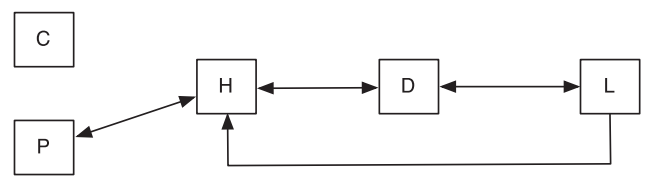

Let $(A$, dom, I $) \in \mathcal{C}_{\mathcal{D G}}$ if and only if $(A$, dom, I $)$ is an interpretation of $\mathcal{D G}$ such that $\mathrm{I}(\mathrm{rel})(\alpha, a)=\left(\mathrm{fta}_{D}^{\hookrightarrow}(\alpha), \alpha\right)$ for all $\alpha \in A^{*}$ and $a \in A$ with $\operatorname{dom}(a)=D$. Thus, architectural interpretations in $\mathcal{C}_{\mathcal{D G}}$ ensure that $\mathrm{fta}_{D} \overleftrightarrow{\prime}^{\prime}$ is an upper bound on information that may be released from $D$ to $L$. It is straightforward to check that these architectural specifications are nonconflating. The required compatibility constraint is also satisfied, as shown in the following result.

Proposition 3.4. If $(A$, dom, $\mathrm{I}) \in \mathcal{C}_{\mathcal{D G}}$, then $\mathrm{I}($ rel $)$ is $\mathrm{fta}_{D}^{\hookrightarrow}$-compatible.

The architectural specification imposes restrictions on what information $L$ knows and when. Domain $L$ can only learn about other domains via downgrader $D$. The restriction on filter functions for rel ensures that $L$ cannot know anything about the actions of $C$, since $C \succ^{\prime} H$, and so the function $\mathrm{fta}_{D}^{\rightleftarrows^{\prime}}$ does not contain any information about the actions of $C$. However, information about the actions of $H, P$, and $D$ may be released to $L$. Additionally, $L$ cannot learn about actions of other domains that occurred after the last $D$ action. The following two propositions express these restrictions.

Proposition 3.5. If $M$ is FTA-compliant with architectural specification $\left(\mathcal{D G}, \mathcal{C}_{\mathcal{D G}}\right)$ and $\pi(p)$ depends on $C$ actions at $\alpha$, then $M, \pi, \alpha \vDash \neg K_{L} p$.

Actions of the downgrader are the only means by which $L$ acquires knowledge about other domains' actions. Thus, $L$ knows nothing about any action of other domains that occur after the last $D$ action. To state this formally, we say that proposition $X$ is about $C, P$, and $H$ activity after the last $D$ action if there exists a set $Y \subseteq A^{*}$ such that for all $\alpha \in A^{*}, \alpha \in X \Longleftrightarrow F(\alpha) \in Y$, where $F$ is defined recursively by $\overline{F(\epsilon)}=\epsilon$ and

$$
F(\beta a)= \begin{cases}\epsilon & \text { if } \operatorname{dom}(a)=D \\ F(\beta) a & \text { if } \operatorname{dom}(a) \in\{C, P, H\} \\ F(\beta) & \text { otherwise. }\end{cases}
$$

Intuitively, $F$ returns the $C, P$, and $H$ actions that occur after the last $D$ action, and so proposition $X$ is about $C, P$, and $H$ activity after the last $D$ action if membership in $X$ depends only on the $C, P$, and $H$ actions that occur after the last $D$ action.

Proposition 3.6. If $M$ is FTA-compliant with architectural specification $\left(\mathcal{D G}, \mathcal{C}_{\mathcal{D G}}\right)$ and $\pi(p)$ is a nontrivial proposition about $C, P$, and $H$ activity after the last $D$ action, then $M, \pi \vDash \neg K_{L} p$.

\subsection{Example: Electronic Election}

Figure 4 shows architecture $\mathcal{E} \mathcal{E}$, an electronic election for $n$ voters, coordinated by an election authority ElecAuth. There is one domain $v_{i}$ for each voter and a single domain ElecAuth, for the election authority. The architecture permits arbitrary information to flow from a voter to the election authority but uses filter function results to restrict what information may flow from the election authority to each voter.

In many elections, the behavior of individual voters is confidential information: it should not be known how voters voted. (Elections have several security requirements. 


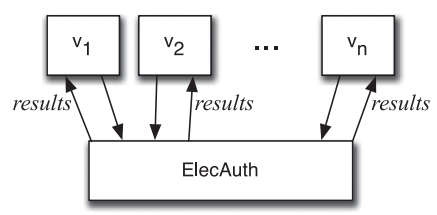

Fig. 4. Architecture $\mathcal{E} \mathcal{E}$.

For example, the final result should be correctly computed from the votes-an integrity requirement. We focus here on the confidentiality requirement for voters.)

By specifying an additional local constraint on the election authority, we can show that this architecture enforces anonymity on the identity of the voters, thus satisfying the confidentiality requirement about the behavior of voters. The election authority's compliance with this local constraint might be assured by means of a careful verification of its implementation or carefully designed cryptographic protocols (which may remove some or all of the trust required to be placed in the election authority).

We first assume that voters are homogenous in that they have the same set of actions. Let $(\mathcal{E} \mathcal{E}, \mathcal{I})$ be an interpreted architecture, where $\mathcal{I}=(A$, dom, $I)$. We say that architecture interpretation $\mathcal{I}$ is voter homogenous if for any voter $v$, the set of possible actions $A_{v}=\{a \in A \mid \operatorname{dom}(a)=v\}$ equals $\left\{a^{v} \mid a \in A_{V}\right\}$, where $A_{V}$ is the set of action types available to voters. Intuitively, $a^{v}$ represent the action of type $a$ when performed by voter $v$.

We define a voter permutation $P$ as a permutation over the set of voters $V$. Since all voters have the same set of actions in a voter-homogenous interpretation, we can apply a permutation $P$ to sequence $\alpha \in A^{*}$, written $P(\alpha)$, as follows:

$$
\begin{aligned}
P(\epsilon) & =\epsilon & & \\
P(\alpha a) & =P(\alpha) a & & \text { if } \operatorname{dom}(a) \notin V \\
P\left(\alpha a^{v}\right) & =P(\alpha) a^{P(v)} & & \text { if } a \in A_{V} .
\end{aligned}
$$

We apply permutation $P$ to proposition $X \subseteq A^{*}$ by applying $P$ to each sequence $\alpha \in X$ (i.e., $P(X)=\{P(\alpha) \mid \alpha \in X\}$ ).

Using voter permutations, we can now state the local constraint that election results do not depend on (and thus do not reveal) the identity of any voter.

A1. Election results have the following identity-oblivious property: given voterhomogenous interpreted architecture $(\mathcal{E} \mathcal{E}, \mathcal{I})$ where $\mathcal{I}=(A$, dom, I), for all voter permutations $P$, sequences $\alpha \in A^{*}$, and actions $a \in A$ with $\operatorname{dom}(a)=$ ElecAuth, we have $\mathrm{I}($ results $)(\alpha, a)=\mathrm{I}($ results $)(P(\alpha), a)$.

There are several possible interpretations of results that satisfy this constraint, such as a function that returns each candidate and her vote tally, or a function that returns the total number of votes submitted. However, a function that returns a ballot and the identity of the voter that submitted it does not satisfy constraint A1.

We define architecture specification $\mathcal{C}_{\mathcal{E E}}$ such that $\mathcal{I} \in \mathcal{C}_{\mathcal{E E}}$ if and only if $\mathcal{I}$ is a voterhomogenous interpretation of $\mathcal{E} \mathcal{E}$ that satisfies constraint $\mathrm{A} 1$.

Given the local constraint A1, we can show that if voter $v$ believes that some proposition $X$ may be satisfied, then $v$ also believes that $P(X)$ may be satisfied, for any voter permutation $P$ with $P(v)=v$. For example, if Alice considers it possible that Bob voted for Obama and Charlie voted for Romney, then Alice considers it possible that Charlie voted for Obama and Bob voted for Romney. 
Proposition 3.7. Let system $M$ be FTA-compliant with architectural specification $\left(\mathcal{E} \mathcal{E}, \mathcal{C}_{\mathcal{E E}}\right)$. Let $v$ be a voter. For all voter permutations $P$ such that $P(v)=v$, if $\pi(q)=$ $P(\pi(p))$, then $M, \pi, \alpha \vDash \neg K_{v} \neg p \Rightarrow \neg K_{v} \neg q$ for all $\alpha \in A^{*}$.

Note that Proposition 3.7 does not imply that voter $v$ learns nothing about other voters. For example, if the election results reveal that all voters voted for Obama, then voter $v$ knows how every other voter voted. However, Proposition 3.7 provides anonymity: given the results, voter $v$ cannot distinguish the behavior of other voters.

\subsection{Group Knowledge Versus Distributed Knowledge}

We note that the notion of group knowledge $K_{G}$ differs from distributed knowledge [Fagin et al. 1995], the notion most commonly used in the literature on epistemic logic for the knowledge that a group would have if they pooled their local information. To provide semantics for distributed knowledge, we define indistinguishability relation $\approx_{G}^{D}$ as the intersection of $\approx_{u}$ for $u \in G$. The distributed knowledge operator $D_{G}$ for group $G$ is given semantics by

$$
M, \pi, \alpha \vDash D_{G} \phi \text { if and only if } M, \pi, \alpha^{\prime} \vDash \phi \text { for all } \alpha^{\prime} \in A^{*} \text { s.t. } \alpha \approx_{G}^{D} \alpha^{\prime} .
$$

We write $\mathcal{L}^{D}(\Phi)$ for the set of the formulas that can be built starting from $\top$ and the primitive propositions in $\Phi$, using conjunction, negation, and modal operators $K_{G}$ and $D_{G}$. In other words, $\mathcal{L}^{D}(\Phi)$ extends $\mathcal{L}(\Phi)$ with the distributed knowledge modal operators $D_{G}$.

The reason we use group knowledge is that it proves to have a stronger relationship to a type of architectural abstraction that we consider next. The two notions are related by the following result.

Lemma 3.8. For $u \in G$, the formulas $K_{u} \phi \Rightarrow D_{G} \phi$ and $D_{G} \phi \Rightarrow K_{G} \phi$ are valid.

The converse relationship $K_{G} \phi \Rightarrow D_{G} \phi$ is not valid. For example, consider a system $M$ with exactly two domains $u, v$, which both make observation $\perp$ at all states. Let $G=\{u, v\}$. Consider the proposition $p$ with $\pi(p)$ consisting of all sequences in which there is an action of domain $u$ that precedes any action of domain $v$. Let $\alpha=a_{u} a_{v}$ and $\alpha^{\prime}=a_{v} a_{u}$, where $a_{u}$ is an action of $u$ and $a_{v}$ is an action of $v$. Then $M, \pi, \alpha \models K_{G} p$, since view $_{G}(\alpha)=$ view $_{G}(\beta)$ implies that $\beta=\alpha$. However, we have view $(\alpha)=\perp a_{u} \perp=$ view $_{u}\left(\alpha^{\prime}\right)$, and similarly for domain $v$, so $\alpha \approx_{G}^{D} \alpha^{\prime}$. Since $M, \pi, \alpha^{\prime} \not \models p$, we obtain that $M, \pi, \alpha \not D_{G} p$. We remark that the example relies upon the assumption of asynchrony: it can be shown that in synchronous systems we have $K_{G} \phi \equiv D_{G} \phi$.

Compared to distributed knowledge $D_{G} \phi$, the less common notion $K_{G} \phi$ of group knowledge captures the way that knowledge properties are preserved under a particular type of architectural abstraction. Given a system $M=\left\langle S, s_{0}, A, D_{1}\right.$, step, obs, dom $\rangle$ and a surjective mapping $r: D_{1} \rightarrow D_{2}$, define $r(M)=\left\langle S, s_{0}, A, D_{2}\right.$, step, obs', dom' $\rangle$ to be the system that is identical to $M$, except that it has domains $D_{2}$, and the functions dom' $^{\prime}$ and obs' are defined by dom' $=r \circ$ dom, and, for $u \in D_{2}, \operatorname{obs}_{u}^{\prime}(s)=\operatorname{obs}_{G}(s)$, where $G=r^{-1}(u)$. Note that machine $r(M)$ is essentially the same as $M$; only the domains and the observation function changes. This contrasts with other refinement notions where the operation of the machine changes under refinement. Intuitively, each domain $u$ in $r(M)$ corresponds to the group of domains $r^{-1}(u)$ in $M$, with every action of a domain in $r^{-1}(u)$ treated as an action of $u$. Similarly, for a formula $\phi$ of the epistemic logic, we write $r^{-1}(\phi)$ for the formula obtained by replacing each occurrence of a group $G$ in a modal operator in $\phi$ by the group $r^{-1}(G)$.

The existence of a surjective mapping $r: D_{1} \rightarrow D_{2}$ is one requirement for architectural refinement, which we discuss in greater detail in Appendix A. The following result shows that abstracting a system $M$ to $r(M)$ (or, conversely, refining $r(M)$ to $M$ ) 
preserves satisfaction of formulas, subject to a corresponding abstraction on groups being applied in the formulas.

Theorem 3.9. Let $r: D_{1} \rightarrow D_{2}$ be surjective and let $M$ be a system with domains $D_{1}$. Then for all interpretations $\pi$, sequences of actions $\alpha$ of $M$, and formulas $\phi \in \mathcal{L}(\Phi)$ for agents $D_{2}$ we have $r(M), \pi, \alpha \models \phi$ if and only if $M, \pi, \alpha \models r^{-1}(\phi)$.

We note that the preceding example to show the difference between group and distributed knowledge also shows that Theorem 3.9 would not hold if we were to include distributed knowledge in the language and analogously define $r^{-1}\left(D_{G} \phi\right)=D_{r^{-1}(G)} \phi$. For example, consider the function $r$ with $r(u)=r(v)=w$. Since $r^{-1}\left(K_{w} p\right)=K_{\{u, v\}} p$ and $M, \pi, \alpha \models K_{\{u, v\}} p$, we have $r(M), \pi, \alpha \models K_{w} p$, and hence $r(M), \pi, \alpha \models D_{w} p$. However, as shown earlier, we do not have $M, \pi, \alpha=D_{\{u, v\}} p$ (i.e., we do not have $M, \pi, \alpha=r^{-1}\left(D_{w} p\right)$ ).

We can also reason about how abstraction affects $G$-dependent propositions.

Lemma 3.10. Let $r: D_{1} \rightarrow D_{2}$ be surjective and let $M$ be a system with domains $D_{1}$. Then proposition $X$ depends on Gactions at $\alpha$ in $r(M)$ if and only if Xdepends on $r^{-1}(G)$ actions at $\alpha$ in $M$.

\section{IMPLEMENTING ARCHITECTURES USING ACCESS CONTROL}

One of the mechanisms that might be used to enforce compliance with an information flow architecture is access control restrictions on the ability of domains to read and write objects. This idea was already implicit in the Bell and LaPadula [1976] approach of enforcing that high-level information should not flow to low-level domains through a "no read up" and "no write down" access control policy. The idea was given a more semantically well-founded expression by Rushby [1992], who established a formal relation between access control systems and a theory of information flow based on intransitive noninterference policies. Rushby's "reference monitor conditions" give semantics to the notion of reading and writing, which was absent in the work of Bell and LaPadula. Rushby's formulation was sharpened and shown to be closely related to TA-security by van der Meyden [2007].

In this section, we present a generalization of van der Meyden's formulation of access control and show how enforcement of an access control policy together with local verification of trusted components can be used to assure that a system is compliant with an extended architecture.

We first recall some definitions and results from van der Meyden [2007]. The system model we have used to this point does not require the states of a system to be equipped with any internal structure. In practice, systems typically will be constructed as an assembly of components. To capture this, Rushby [1992] introduced the notion of a system with structured state, which is a system $M$ (with states $S$ and domains $D$ ) together with

(1) a set $O b j$ of objects,

(2) a set $V$ of values, and functions

(3) contents : $S \times \mathrm{Obj} \rightarrow V$, with $\operatorname{contents}(s, n)$ interpreted as the value of object $n$ in state $s$, and

(4) observe, alter $: D \rightarrow \mathcal{P}(\mathrm{Obj})$, with observe $(u)$ and alter $(u)$ interpreted as the set of objects that domain $u$ can observe (or read) and alter (or write), respectively.

For brevity, we write $s(x)$ for contents $(s, x)$. We call the pair (observe, alter) the access control table of the machine. For each domain $u$, we define an equivalence relation of "observable content equivalence" on states $s, t \in S$ by $s \approx_{u}^{o c} t$ if $s(x)=t(x)$ for all $x \in$ observe $(u)$. 
Rushby introduced reference monitor conditions on such machines to capture formally the intuitions associated with the pair (observe, alter) being an access control table that restricts the ability of the actions to "read" and "write" the objects Obj. Van der Meyden [2007] sharpened these conditions to the following (the difference is in RM2):

RM1. If $s \approx_{u}^{o c} t$, then $\mathrm{obs}_{u}(s)=\mathrm{obs}_{u}(t)$.

RM2. For all actions $a \in A$, states $s, t \in S$, and objects $x \in \operatorname{alter}(\operatorname{dom}(a))$, if $s \approx_{\operatorname{dom}(a)}^{o c} t$ and $s(x)=t(x)$, then $(s \cdot a)(x)=(t \cdot a)(x)$.

RM3. If $x \notin$ alter $(\operatorname{dom}(a))$, then $s(x)=(s \cdot a)(x)$.

Intuitively, RM1 states that a domain's observation depends only on the values of the objects that it can observe (or read). RM2 states that if action $a$ is performed in a domain $u$ that is permitted to alter an object $x$, then the new value of the object after the action depends only on its old value and the values of objects that domain $u$ is permitted to observe. The final condition RM3 says that if action $a$ is performed in a domain that is not permitted to alter (or write) an object $x$, then the value of $x$ does not change.

We note that the terminology "reference monitor conditions" points to the fact that these conditions can be enforced by a reference monitor that mediates all attempts to perform an action, simply by denying requests by a domain $u$ to read an object not in observe $(u)$ or write to an object not in alter $(u)$.

In addition to the reference monitor assumptions, Rushby considers a condition ${ }^{4}$ stating that if there is an object that may be altered by domain $u$ and observed by domain $v$, then the information flow policy should permit flow of information from $u$ to $v$. (Obviously, the object $x$ provides a channel for information to flow from $u$ to $v$.)

AOI. If alter $(u) \cap \operatorname{observe}(v) \neq \emptyset$, then $u \longmapsto v$.

Van der Meyden [2007] shows the following, strengthening a result of Rushby [1992].

THEOREM 4.1. If $M$ is a system with structured state satisfying RM1 through RM3 and AOI with respect to $\longmapsto$, then $M$ is TA-secure with respect to $\longmapsto$.

We now develop a generalization of this result to extended architectures. As a first step, note that in extended architectures, the situation where the information flow policy potentially permits flow of information from domain $u$ to domain $v$ corresponds to the existence of an edge $u \stackrel{f}{\rightarrow} v$ for some label $f$ (possibly $T$ ). This motivates the following variant of condition AOI:

$\mathrm{AOI}^{\prime}$. If alter $(u) \cap \operatorname{observe}(v) \neq \emptyset$, then $u_{\downarrow} f \underset{v}{ }$ for some $f$.

Next we develop a set of conditions that check that information flow constraints of the form $u \stackrel{f}{\rightarrow} v$ with $f \neq \top$ have been correctly implemented in a system. Let $\mathcal{I}=(A, \operatorname{dom}, \mathrm{I})$ be an interpretation of architecture $\mathcal{A}=(D, \longmapsto)$. Consider the following constraints in a system $M$ with actions $A$, domains $D$, and domain function dom:

I1. If $\operatorname{dom}(a) \underset{f}{f} u$ for $f \neq \top$ and $\mathrm{I}(f)(\alpha, a)=\epsilon$ and $x \in$ observe $(u) \cap \operatorname{alter}(\operatorname{dom}(a))$, then $\left(s_{0} \cdot \alpha a\right)(x)=\left(s_{0} \cdot \alpha\right)(x)$.

I2. If $\operatorname{dom}(a) \underset{\downarrow}{\rightarrow} u$ with $f \neq \top$ and $\operatorname{dom}(b) \stackrel{g}{\rightarrow} u$ with $f \neq \top$ and $\mathrm{I}(f)(\alpha, a)=\mathrm{I}(g)(\beta, b) \neq \epsilon$ and $x \in$ observe $(u) \cap(\operatorname{alter}(\operatorname{dom}(a)) \cup \operatorname{alter}(\operatorname{dom}(b)))$ and $\left(s_{0} \cdot \alpha\right)(x)=\left(s_{0} \cdot \beta\right)(x)$, then $\left(s_{0} \cdot \alpha a\right)(x)=\left(s_{0} \cdot \beta b\right)(x)$.

\footnotetext{
4"AOI" derives from the fact that this condition connects the "alter," "observe," and "interferes" constructs.
} 
Condition I1 ensures that if filter function $f$ restricts how the domain of action $a$ may interact with domain $u$ (i.e., $\operatorname{dom}(a) \stackrel{f}{\rightarrow} u$ with $f \neq \top$ ), and the filter function interpretation does not allow any information flow $\operatorname{I}(f)(\alpha, a)=\epsilon)$, then the action does not change the state of any object $x$ that domain $u$ may observe and domain $\operatorname{dom}(a)$ is allowed to alter. Condition I2 states that if an action $a$ in domain $\operatorname{dom}(a)$ may alter an object $x$ that is observable by domain $u$ and $\operatorname{dom}(a) \stackrel{f}{\rightarrow} u$, then the new state of object $x$ is restricted by what information flow is permitted by the interpretation of $f$. Specifically, if $\mathrm{I}(f)(\alpha, a)=\mathrm{I}(g)(\beta, b)$ (for sequences $\alpha, \beta \in A^{*}$ and actions $a, b \in A$ ), then the information revealed by $a$ in trace $\alpha$ should be identical to the information revealed by $b$ in trace $\beta$, and so the contents of object $x$ must be identical in trace $\alpha a$ and $\beta b$.

We note that verification of these constraints requires consideration only of domains that are trusted, in the sense that they have outgoing edges not labeled $T$, and the objects that such domains are permitted to alter. Thus, verification of these constraints can be localized to the trusted domains. The following result states that such local verification, together with enforcement of an access control policy consistent with the information flow policy via a mechanism satisfying the reference monitor constraints, suffices to assure that an information flow policy has been satisfied.

TheOREM 4.2. Let $\mathcal{A I}$ be a strongly nonconflating interpreted architecture. Suppose that $M$ is a system with structured state satisfying $R M 1$ through $R M 3, A O I^{\prime}$, and II and I2. Then $M$ is FTA-compliant with $\mathcal{A} I$.

Conditions I1 and I2 are still somewhat nonlocal since both refer to $\alpha$ and I2 also refers to $\beta$. Since architectural specifications are stated in terms of these state sequences, references to them cannot be completely eliminated. However, it is often convenient to factor this reference via properties of the state of the system. For every edge label $f$, let $F(f)$ be a function with domain $S \times A$. Suppose that these functions satisfy the following conditions for all $f, g, \alpha, \beta, a$ and $b$.

E1. I $(f)(\alpha, a)=\mathrm{I}(g)(\beta, b)$ implies that $F(f)\left(s_{0} \cdot \alpha, a\right)=F(g)\left(s_{0} \cdot \beta, b\right)$

E2. I $(f)(\alpha, a)=\epsilon$ implies that $F(f)\left(s_{0} \cdot \alpha, a\right)=\epsilon$.

Intuitively, $F$ gives a state-based encoding of an approximation to I. (In general, the value $F\left(s_{0} \cdot \alpha, a\right)$ may convey less information than $\mathrm{I}(f)(\alpha, a)$. When $\mathrm{I}(f)(\alpha, a)=\epsilon$, representing that no information is conveyed, $F\left(s_{0} \cdot \alpha, a\right)$ also equals $\epsilon$, similarly representing that no information is conveyed.)

The following conditions use $F$ to give a variant of the conditions I1 and I 2 that is stated with respect to the states of the machine:

I1'. If $\operatorname{dom}(a)_{\downarrow} f \underset{u}{ } u$ for $f \neq \top$ and $F(f)(s, a)=\epsilon$ and $x \in$ observe $(u) \cap \operatorname{alter}(\operatorname{dom}(a))$, then $(s \cdot a)(x)=(s)(x)$.

I2'. If $\operatorname{dom}(a) \stackrel{f}{\rightarrow} u$ with $f \neq \top \operatorname{dom}(b) \stackrel{g}{g} u$ with $g \neq \top$ and $F(f)(s, a)=F(g)(t, b) \neq \epsilon$ and $x \in$ observe $(u) \cap(\operatorname{alter}(\operatorname{dom}(a)) \cup \operatorname{alter}(\operatorname{dom}(b)))$ and $s(x)=t(x)$, then $(s \cdot a)(x)=$ $(t \cdot b)(x)$.

This state-factored representation implies conditions I1 and I2.

\section{Theorem 4.3. Conditions E1 and E2 and I1' and I2' imply I1 and I2.}

To illustrate the application of these results, we consider the examples of architectural specifications introduced earlier and show how some particular systems may be proved to be implementations of these specifications. 


\subsection{Starlight Architecture}

We present an implementation of the Starlight architectural specification $\left(\mathcal{S L}, \mathcal{C}_{\mathcal{S} \mathcal{L}}\right)$ as a system with structured state. We first select an architectural interpretation $\mathcal{I}=$ $(A$, dom, $I)$ in $\mathcal{C}_{\mathcal{S L}}$. The actions $A$ of the interpretation are given with their associated domain as follows:

- The actions of domain $S$ consist of the toggle action $t$, plus actions $k$ from a set $\mathcal{K}$, which intuitively represents the set of keyboard actions.

- The actions of domain $H$ consist of the action get, plus actions $h$ drawn from some set $E_{H}$ representing possible high-level events.

-The actions of domain $L$ consist of the action put, plus actions $l$ drawn from some set $E_{L}$ representing the possible low-level events.

As required by $\mathcal{C}_{\mathcal{S L}}$, we assume that I satisfies

$$
\mathrm{I}(s f)(\alpha, a)= \begin{cases}a & \text { if } a=\mathrm{t} \text { or } \\ & \#_{\mathrm{t}}(\alpha) \text { is odd and } a \in A_{S} \\ \epsilon & \text { otherwise, }\end{cases}
$$

where $\#_{\mathrm{t}}(\alpha)$ is the number of occurrences of $t$ in $\alpha$.

Next we construct a system $M$ for the interpreted architecture $(\mathcal{S} \mathcal{L}, \mathcal{I})$. The system $M$ is based on the set of objects $\mathrm{Obj}=\{\log H, \log L, \operatorname{tog} p o s\}$. Intuitively, $\log H$ and $\log L$ are logs of actions observable to $H$ and $L$, respectively, and togpos indicates the position of the toggle switch. The objects $\log H$ and $\log L$ have values in $A^{*}$, and togpos has a value in $\{H, L\}$. A state $s$ is just an assignment of value of the given type to each of the objects, and we have contents $(s, x)=s(x)$. The initial state $s_{0}$ is defined by $s(\log H)=s(\log L)=\epsilon$ and $s(\operatorname{tog} p o s)=H$. Transitions are defined by the following code associated to each of the actions:

-for domain $S$ :

$$
\begin{aligned}
& \mathrm{t}: \text { if togpos }=H, \text { then togpos }:=L \text { else togpos }:=H \\
& k: \text { if togpos }=H, \text { then } \log H:=\log H^{\wedge} k \text { else } \log L:=\log L^{\wedge} k
\end{aligned}
$$

In other words, the toggle action $t$ changes the position of the toggle, and $k$ is recorded in either the high level log or the low level log, depending on the position of the toggle. -for domain $H$ :

$$
\begin{aligned}
& h: \log H:=\log H^{\wedge} h \\
& \text { get: } \log H:=\log H^{\wedge} \log L
\end{aligned}
$$

Thus, the events $h$ are recorded in the high level log, and get fetches a copy of the low level log.

-for domain $L$ :

$$
\begin{aligned}
& l: \log L:=\log L^{\wedge} l \\
& \text { put: } \log H:=\log H^{\wedge} \log L
\end{aligned}
$$

Similarly, the events $l$ are recorded in the low level log, and put pushes a copy of the low level log into the high level log.

The access control functions alter and observe are defined by the following access control table. Here, for an object $x$ and a domain $u$, we have an entry $a$ (or $o$ ) in the

\begin{tabular}{|c||c|c|c|}
\hline & $H$ & $L$ & $S$ \\
\hline $\log H$ & $\mathrm{a}, \mathrm{o}$ & $\mathrm{a}$ & $\mathrm{a}$ \\
$\log L$ & $\mathrm{o}$ & $\mathrm{a}, \mathrm{o}$ & $\mathrm{a}$ \\
togpos & & & $\mathrm{a}, \mathrm{o}$ \\
\hline
\end{tabular}

corresponding cell of the table just when $x \in \operatorname{alter}(u)$ (respectively, $x \in$ observe $(u)$ ). We define observations in the system by allowing each domain to observe the values 
of all of its observable objects. In other words, for each domain $u$ and state $s$, we define $\operatorname{obs}_{u}(s)=s\lceil\operatorname{observe}(u)$.

Proposition 4.4. The system $M$ is FTA-compliant with architectural specification $\left(\mathcal{S} \mathcal{L}, \mathcal{C}_{\mathcal{S L}}\right)$

\subsection{Electronic Election}

We describe a system that implements the architectural specification $\left(\mathcal{E} \mathcal{E}, \mathcal{C}_{\mathcal{E}}\right)$ for an electronic election of Section 3.5.

We first select a particular architectural interpretation $\mathcal{I}=(A$, dom, $I)$ that satisfies this specification. We suppose that the election is a referendum with the voters voting either "yes" or "no" and the decision determined by a majority of the voters. We take the set of domains to be $V \cup\{$ Elec Auth $\}$, where $V=\left\{V_{1}, \ldots, V_{n}\right\}$ represents the set of voters, and Elec Auth, is the election authority. The set of actions $A=\left\{\operatorname{yes}^{v} \mid v \in V\right\} \cup\left\{\right.$ nov $^{v} \mid v \in$ $V\} \cup\left\{\right.$ tally , with associated domains given by $\operatorname{dom}\left(\operatorname{yes}^{v}\right)=\operatorname{dom}\left(\right.$ no $\left.^{v}\right)=v$ for all $v \in V$ and $\operatorname{dom}(\mathrm{tally})=$ Elec Auth. Note that these actions are voter homogeneous, as required by $\mathcal{C}_{\mathcal{E E}}$.

The architecture has just one edge label, results. Thus, for the interpretation I, we need to define I(results). Given a sequence $\alpha$, define the latest action of voter $v$ to be the action $a \in\left\{\right.$ yes $^{v}$, nov $\left.^{v}\right\}$ such that $\alpha=\alpha_{0} a \alpha_{1}$ and $\alpha_{1}$ contains no action $b$ with $\operatorname{dom}(b)=v$, if such a decomposition exists, or $\perp$ otherwise. We now define I(results)( $\alpha$, tally) for $\alpha \in A^{*}$ to be the number of voters $v$ whose latest action is yes ${ }^{v}$. (Since the election authority has only the one action tally, this is all that is required to specify I.) In other words, in this interpretation, the information that the election authority is permitted to reveal is the number of voters who have voted yes in the latest round of voting. (We assume that a round consists of the events between two consecutive tally actions, but that if a voter does not vote in round, his or her vote defaults to that voter's vote in a previous round, if any.) It is easily seen that this interpretation is identity oblivious. Thus, $\mathcal{I} \in \mathcal{C}_{\mathcal{E E}}$.

Next, we describe a system $M$ with structured state and argue that it FTA-complies with interpreted architecture $(\mathcal{E} \mathcal{E}, \mathcal{I})$. We take the set of objects of $M$ to be the set $\mathrm{Obj}=\left\{v_{1}, \ldots, v_{n}, b b\right\}$, where $v_{i}$ represents the election authority's record of the vote of voter $i$ and $b$ represents a bulletin board where the results of the election are broadcast to the voters. The objects $v_{i}$ and $b b$ take a value in $\{\perp, Y, N\}$, with $\perp$ indicating that no vote has yet been made by the voter, or in the case of $b b$, that the election authority has not yet announced a result. In the initial state, all objects take value $\perp$.

Access control on these objects is captured by the following table:

\begin{tabular}{|c|c|c|}
\hline & $V_{i}$ & Elec Auth \\
\hline observe & $b b$ & $v_{1}, \ldots, v_{n}, b b$ \\
\hline alter & $v_{i}$ & $b b$ \\
\hline
\end{tabular}

It is straightforward to verify that this table satisfies condition $\mathrm{AOI}^{\prime}$ for policy $\mathcal{E} \mathcal{E}$. We let each domain's observation consist of the values of its observable objects (i.e., $\operatorname{obs}_{u}(s)=s\lceil$ observe $(u))$ so that RM1 is satisfied trivially.

The effect of the actions on the state is given by the following code:

$$
\begin{aligned}
& \text { yes } V_{i}: v_{i}:=Y \\
& \text { no } V_{i}: v_{i}:=N \\
& \text { tally }: \text { if }\left|\left\{i \mid v_{i}=Y\right\}\right| \geq n / 2 \text {, then } b b:=Y \text { else } b b:=N .
\end{aligned}
$$

These definitions satisfy conditions RM2 and RM3. For RM2, note that the actions yes $^{V_{i}}$ and no ${ }^{V_{i}}$ change $v_{i}$ in a way that depends only on the action. The action tally 
changes $b$ in a way that depends on $v_{1} \ldots, v_{n}$, but all of these objects are observable to Elec Auth. For RM3, we have that the complement of alter $\left(V_{i}\right)$ is $\operatorname{Obj} \backslash\left\{v_{i}\right\}$, but $V_{i}$ 's actions change only $v_{i}$, and the complement of alter(ElecAuth) is $\mathrm{Obj} \backslash\{b b\}$, and none of the $v_{i}$ are changed by Elec Auth's action tally.

To show that this system FTA-complies with the interpreted architecture $(\mathcal{E} \mathcal{E}, \mathcal{I})$, we use conditions $\mathrm{E} 1$ and $\mathrm{E} 2$ and $\mathrm{I} 1^{\prime}$ and $\mathrm{I} 2^{\prime}$. Let the functions $F$ be given by $F($ results $)(s$, tally $)=\left(\left|\left\{i \mid s\left(v_{i}\right)=Y\right\}\right| \geq n / 2\right)$ (i.e., the output of $F$ is a Boolean value that indicates whether the majority of the $v_{i}$ have value $Y$ ).

We first show that these satisfy E1 and E2. Condition E2 is trivial, since it is never the case that $F$ (results $)(s, a)=\epsilon$. For E1, we first claim that for all sequences $\alpha \in A^{*}$, we have that $\left(s_{0} \cdot \alpha\right)\left(v_{i}\right)=Y$ if and only if the latest action of $V_{i}$ in $\alpha$ is yes. The proof of this benefits from RM3: since $v_{i} \in \operatorname{alter}(\operatorname{dom}(a))$ if and only if $\operatorname{dom}(a)=V_{i}$, we have by a straightforward induction that $\left(s_{0} \cdot \alpha\right)\left(v_{i}\right)=\left(s_{0} \cdot \alpha \uparrow\left\{V_{i}\right\}\right)\left(v_{i}\right)$. The latest action of domain $V_{i}$ in $\alpha$ is the final action (if any) in $\alpha \uparrow\left\{V_{i}\right\}$, and $\left(s_{0} \cdot \alpha \uparrow\left\{V_{i}\right\}\right)\left(v_{i}\right)=Y$ just in case this action exists and equals yes. The claim now follows.

We now verify the conditions $\mathrm{I}^{\prime}$ and $\mathrm{I} 2^{\prime}$. Condition $\mathrm{I}^{\prime}{ }^{\prime}$ is trivial since we never have $F($ results $)(s$, tally $)=\epsilon$. For condition I2', suppose that $\operatorname{dom}(a) \stackrel{f}{\rightarrow} u$ with $f \neq \top$ and $\operatorname{dom}(b), g$ with $g \neq \top$. Then we must have $f=g=\operatorname{results}$ and $\operatorname{dom}(a)=$ ElecAuth, hence $a=$ tally and $u=V_{i}$ for some $i$. Suppose additionally that $F(f)(s, a)=F(g)(t, b) \neq \epsilon$ and $x \in \operatorname{observe}(u) \cap(\operatorname{alter}(\operatorname{dom}(a)) \cup$ alter $(\operatorname{dom}(b)))$ and $s(x)=t(x)$. Then $x \in$ observe $\left(V_{i}\right) \cap$ alter(ElecAuth), so $x=b b$. By definition of $F$, we have $F($ results $)(s$, tally $)=\left(\left|\left\{i \mid s\left(v_{i}\right)=Y\right\}\right| \geq n / 2\right)=\left(\left|\left\{i \mid t\left(v_{i}\right)=Y\right\}\right| \geq n / 2\right)=$ $F($ results $)(t$, tally). In case this Boolean value is true, we have $(s \cdot a)(x)=Y=(t \cdot a)(x)$, otherwise $(s \cdot a)(x)=N=(t \cdot a)(x)$. In either case, $(s \cdot a)(x)=(t \cdot a)(x)$, as required.

We remark that in this argument, we have used a function $F$ (results) that is not an equivalent state-based encoding of the interpretation I(results) but which is weaker than this interpretation. Correspondingly, in the implementation, the election authority reveals less information to the voters than the architectural interpretation permits. The architectural interpretation permits the election authority to reveal the number of voters who have voted "yes," but in the implementation the election authority only reveals whether this number is at least $n / 2$. The notion of FTA-compliance with an architecture allows this kind of weakening of information flows in the implementation. We do this to illustrate weakening, but of course it is undesirable in a real election, where the election authority will most likely be required to reveal the actual numbers.

\section{ENFORCING ARCHITECTURES ON CONCRETE PLATFORMS}

The access control model presented in Section 4 gives an abstract view of how an extended architecture might be enforced. It identifies a set of conditions that suffice to ensure that a concrete system complies with the architecture. The process for verification of these conditions in specific settings is likely to depend on the particulars of the implementation platform(s). We briefly discuss a few of the possible platforms and the techniques that might be used to show that the access control conditions hold.

Boettcher et al. [2008] survey techniques to separate components (i.e., to ensure that communication between components is in accordance with the architecture). For unextended architectures, a common technique to ensure architectural compliance is to map information flow edges to physical causality and use physical separation where there is no edge. Thus, the architecture $\mathcal{H L}$ is commonly enforced in military settings by mapping $H$ and $L$ to distinct processors and/or networks and using trusted devices (data diodes) to ensure a one-way information flow from $L$ to $H$. The Starlight Interactive Link [Anderson et al. 1996] is a trusted device that can be added to such an implementation to extend it to an implementation of the architecture $\mathcal{S} \mathcal{L}$. 
One of the long-standing objectives of research on military-grade security has been to avoid the redundancy and consequent expense of such physical implementations, through the use of implementations that enable different security levels to share resources such as memory, processors, and networks. An implementation technique that forms the basis for much work in MILS security is the use of separation kernels, which are highly simplified operating systems with the sole functionality of enforcing an information flow policy. Use of separations kernels introduces the risk that there are covert channels, but much progress has been made in recent years toward formal proofs that separation kernels enforce an information flow policy (e.g., Greve et al. [2003], Heitmeyer et al. [2006], and Murray et al. [2013]).

The key mechanism used to achieve this is typically an access control policy enforced by careful management of hardware access control settings and processor modes to ensure that when a process runs, it may read and write only memory regions authorized by the access control policy. Attempts to read or write memory regions that violate the policy are denied by the hardware access control measures. This ensures that the reference monitor conditions RM2 and RM3 are satisfied. RM1 could be guaranteed by ensuring that all peripheral devices with which a user may interact are mapped to memory regions that are associated to the domain of that user. Together with a check of AOI, this ensures that there are no covert channels exploitable by an asynchronous attacker with respect to the unfiltered architecture. Timing-based covert channels may remain and may be addressed by defenses such as fixed schedules and preventing access to the system clock.

An alternative to the use of hardware access control to ensure satisfaction of the reference monitor conditions is to verify (e.g., using static analysis methods) that the code in each domain (e.g., the code describing how the actions of the domain affect the state) reads and writes only locations that are permitted by the policy. Once this has been done, this code can safely run free of hardware access control. This approach is taken in the Singularity system [Hunt and Larus 2007].

The specifics of the static analysis techniques to be used to enforce the reference monitor conditions will be very language dependent but can benefit from programming language techniques including type safety and encapsulation constructs including objects, object ownership, and aspects [Kiczales 1996]. Some of the abstract reasoning in the preceding examples is already suggestive of such techniques. For example, in the election example, the election authority can be viewed as owning the objects $v_{i}$ and voter $V_{i}$ can be viewed as having a capability to call a method on object $v_{i}$. The code pattern used in the downgrader example for actions $a \in A_{T}$ could be enforced using aspect-oriented techniques.

Once a basic (unextended) architecture has been shown to be enforced by the implementation, it remains to demonstrate that the trusted components in the architecture satisfy their local constraints (e.g., constraints I1 and I2). As these local constraints are application specific, and implementation dependent, it seems unlikely that a single methodology will suffice. We expect that theorem proving, model checking, and language-based information flow techniques [Sabelfeld and Myers 2003] may all be used to provide assurance of satisfaction of the local constraints and the filtering requirements introduced by our extended architectures.

We note that our framework is highly expressive, and it may not always be possible to show compliance with a filtering requirement using only local information. For example, directly implementing the filtering requirements of the downgrader architectural specification $\left(\mathcal{D G}, \mathcal{C}_{\mathcal{D G}}\right)$ by means of controls at the downgrader component $D$ would seem to require the cooperation of high-level components (e.g., provision of secure provenance information) to ensure that the downgrader does not release information concerning $C$. In Appendix A, we develop a refined architectural specification for 
the downgrader that avoids the need for this cooperation by shifting the trust boundary. We also develop an access control implementation of this refined architecture that illustrates how constraints on code can play a role in enforcing an architectural specification. Identifying sufficient conditions for local verification of compliance is an interesting topic for future research.

\section{RELATED WORK}

The most closely related work is that of van der Meyden [2007, 2012], who defines TA-security and considers refinement of architectures on the basis of this semantics. Our contribution is to show that the definition of TA-security supports derivation of global information security properties to extend TA-security to architectures that include filter functions (and, in Appendix A, to develop an account of architectural refinement for these extended architectures). The extension provides a way to specify the behavior of trusted components in a system: intuitively, if a component is the source of an edge in the architecture labeled by a filter function, then the component is trusted to ensure that the interpretation of the filter function limits the information that may flow along the edge. We have presented several examples showing that interesting information-theoretic global security properties can be derived from the very abstract statement that a system complies with such an extended architecture and a set of additional local properties. We note that these global security properties are application specific, capturing more of the unique requirements of applications than the generic property "Low does not know any High secrets" that is most often considered in the literature. We believe that the ability to derive application-specific security properties improves the usefulness and applicability of our approach.

The extended architectures of the present article place upper bounds on the flow of information through a system. Elsewhere [Chong and van der Meyden 2009], we have developed an even richer format that also enables lower bounds on information flow to be expressed. The richer format can express that certain components in the system synchronously observe the state of other components. We demonstrate that this enables architectures to enforce application constraints such as "the results of the auction become known to all bidders at the same time."

Bytschkow et al. [2014] draw on an earlier version of the present article and consider a process algebraic framework with respect to policies with filter functions. Instead of the TA-security style of semantics, they use a standard purge function. In addition to rederiving our Starlight switch example, they consider an example motivated from smart power grids.

The MILS community has identified several application domains that have potential to benefit from MILS architectures and has proposed several concrete architectural instances. These include applications in automotive systems [Camek et al. 2013; Wasicek and Mair 2013], aerospace systems including avionics [Mueller et al. 2012], and air traffic control [Kampichler and Eier 2014]. Generally, these examples are not presented at the level of formal detail that we have attempted in the present work, but they would provide interesting case studies for future research.

Recent work by Guttman and Rowe [2014] considers the propagation of information in distributed systems, regarding the distributed system as a graph connected by unidirectional communication channels. They primarily focus on static models of communication, which are thus similar to architectures that describe permitted communication between components but also consider a dynamic setting, where channel endpoints may be communicated from one component to another and thus new connections between components established during execution. They note that if some cut set separates a source and a sink, then information disclosure from the source to the cut set bounds the information disclosure from the source to the sink. They use 
blur operators to, in effect, describe executions of the system that are observationally indistinguishable and thus to define what information is disclosed.

Other work has sought to formally describe system architecture (e.g., AADL [2009] and Acme [Garlan et al. 2000]) and reason about properties of systems that conform to a given architecture. There are many software engineering concerns that can be reasoned about in architectural design, such as maintainability and reliability. This work focuses on reasoning about the information security of systems, and, as such, our architectures specify local constraints on information that may be communicated between components. The local constraints allow the inference of global information security properties. This work is complementary to work on other aspects of system design.

Relatively little theoretical work takes an architectural perspective on information security. One line of work [Hansson et al. 2008] that takes a similar perspective to ours is conducted in the context the architectural modeling framework AADL. This work is based on the Bell-La Padula model [Bell and LaPadula 1976]. In a similar spirit are works on model-driven security, which extend UML with security modeling notations. Basin et al. [2006] focus on a UML extension for role-based access control policies and model transformations to implementation infrastructures such as Enterprise Java Beans or .NET. Jürjens [2005] extends UML with a focus on reasoning about secrecy in distributed applications employing security protocols. None of these approaches use the application-specific abstract noninterference-style semantics that underpins our contribution, nor do they target the type of reasoning envisaged in the MILS community for development of high-assurance systems built on infrastructure such as separation kernels.

Preservation of information flow properties under composition is problematic. In general, the composition of two secure systems is not guaranteed to be secure. The reason is essentially the same as for refinement: composition reduces the set of possible behaviors of a system, enabling observers to make additional deductions.

Several approaches have been developed that allow security of a composed system to be derived from security of its components. These include use of a stronger definitions of security such as restrictiveness [McCullough 1990] or bisimulation-based nondeducibility on compositions [Focardi and Gorrieri 1994]. McLean [1996] proposes a framework for specifying and reasoning about system composition and the preservation of possibilistic security properties. In this work, we do not focus on showing that security properties of components hold under composition. Instead, we are concerned with proving global security properties and identifying local constraints that components must satisfy. In addition, the literature on preservation of information flow security under composition has largely limited itself to the simple policy $L \longmapsto H$.

Process algebraic operations can be viewed as expressing architectural structure. For example, one could take the view that a process constructed as the parallel composition of two processes $P$ and $Q$, with actions $A$ of the composition then hidden, corresponds to an architecture that $P$ and $Q$ are permitted to interact, but the environment is not permitted to influence through the set of actions $A$. However, the semantics of these operators usually do not preserve this view: typically, this composition is understood in terms of its possible behaviors with respect to the actions that have not been hidden, and the fact that the system has been composed out of two components permitted to interact in a particular way is lost in the meaning of the composition.

Downgrading has historically been one of the motivations for generalizing the notion of noninterference to intransitive policies. Roscoe and Goldsmith [1999] argued against the ipurge-based semantics for noninterference because the meaning it gives to the downgrader policy $H \longmapsto D \longmapsto L$ is too permissive. According to this semantics, any action by the downgrader $D$ "opens the floodgates," since it allows all information 
about the high-security domain $H$ to flow to the low-security domain $L$. Roscoe and Goldsmith proposed to deal with this issue by making the semantics of intransitive noninterference significantly more restrictive, effectively reverting to the purge-based definition of Goguen and Meseguer [1982, 1984]. Our approach to downgrading, using a filter function, provides an alternative approach that enables explicit specification of the information permitted to be released by the downgrader.

More recent work on downgrading has concentrated on downgrading in the setting of language-based security. Sabelfeld and Sands [2005] briefly survey recent work on downgrading in language-based settings and propose several dimensions of downgrading, as well as prudent principles for downgrading. They regard intransitive noninterference as specifying where (in the security levels) downgrading may occur. Since we interpret security levels as system components, our architectures specify where in the system downgrading may occur. The filter functions that we propose in this work specify what information can be downgraded and when this may occur. Thus, our work combines the what, where, and when dimensions of downgrading. Recent work also considers multiple dimensions of downgrading, including Barthe et al. [2008], Banerjee et al. [2008], Mantel and Reinhard [2007], Askarov and Sabelfeld [2007b], and Lux and Mantel [2009]. Motivated specifically from MILS development efforts, Amtoft et al. [2008, 2012] developed an approach to using a specialized Hoare logic to reason about conditional information flow contracts for single components. This is likely to be useful for some instances of our approach but does not handle our architectures in general, which imply conditional information flow constraints that span multiple components.

Recent work [Askarov and Sabelfeld 2007a; Banerjee et al. 2008; Askarov and Myers 2010; Askarov and Chong 2012] considers knowledge-based approaches to downgrading in language-based settings. However, that work does not reason about security properties as general and application specific as in this paper. O'Neill [2006] uses epistemic logic to specify many information security properties but does not directly consider downgrading.

Additional work related to architectural refinement is discussed in Appendix A.6.

\section{CONCLUSION}

Through the examination of several examples, we have shown that strong information security properties can be proven about a system from a high-level architectural description of the system. Any system that complies with the architecture will satisfy the information security properties that can be proven about the architecture.

We extended the notion of system architecture to allow finer-grain specification of what information may be sent between components. This enables the proof of stronger security properties while continuing to provide the benefits of using a high-level architectural description. We generalized the notion of architectural refinement [van der Meyden 2012] for the extended architectures. Certain security properties are preserved by architectural refinement.

The MILS vision is to build high-assurance systems with well-understood security properties by composition of COTS infrastructure and trusted components. This work brings us closer to that goal by demonstrating that it is possible to compositionally derive strong, application-specific, information flow security properties from high-level system specifications.

Many issues remain for future work. The semantics that we have considered here is asynchronous. Although appropriate during high-level design, before mappings to concrete platforms and scheduling decisions have been made, it does not cover attacks based on observations of the timing of events. It would be of interest to develop similar results for a richer timed model. We have expressed constraints in architectural specifications informally, and it would be beneficial to have a formal language to make 
this more rigorous. The enforcement of integrity policies through architectures is also of interest. Finally, giving a formal account of the range of implementation strategies discussed in Section 5 presents many challenges for future efforts.

\section{REFERENCES}

AADL 2009. Architecture Analysis and Design Language (AADL). SAE Standard AS5506/A.

Jim Alves-Foss, W. Scott Harrison, Paul Oman, and Carol Taylor. 2006. The MILS architecture for highassurance embedded systems. International Journal of Embedded Systems 2, 3/4, 239-247.

Torben Amtoft, Josiah Dodds, Zhi Zhang, Andrew W. Appel, Lennart Beringer, John Hatcliff, Xinming $\mathrm{Ou}$, and Andrew Cousino. 2012. A certificate infrastructure for machine-checked proofs of conditional information flow. In Proceedings of the Conference on Principles of Security and Trust. 369-389.

Torben Amtoft, John Hatcliff, Edwin Rodríguez, Robby, Jonathan Hoag, and David Greve. 2008. Specification and checking of software contracts for conditional information flow. In Proceedings of the 15th International Symposium on Formal Methods. 229-245.

Mark Anderson, Chris North, John Griffin, Robert Milner, John Yesberg, and Kenneth Yiu. 1996. Starlight: Interactive link. In Proceedings of the Annual Computer Security Applications Conference. 55-63.

Aslan Askarov and Stephen Chong. 2012. Learning is change in knowledge: Knowledge-based security for dynamic policies. In Proceedings of the 25th IEEE Computer Security Foundations Symposium. IEEE, Los Alamitos, CA, 308-322.

Aslan Askarov and Andrew Myers. 2010. A semantic framework for declassification and endorsement. In Proceedings of the 19th European Symposium on Programming.

Aslan Askarov and Andrei Sabelfeld. 2007a. Gradual release: Unifying declassification, encryption and key release policies. In Proceedings of the IEEE Symposium on Security and Privacy. IEEE, Los Alamitos, CA, 207-221.

Aslan Askarov and Andrei Sabelfeld. 2007b. Localized delimited release: Combining the what and where dimensions of information release. In Proceedings of the 2007 Workshop on Programming Languages and Analysis for Security. ACM, New York, NY, 53-60.

Anindya Banerjee, David A. Naumann, and Stan Rosenberg. 2008. Expressive declassification policies and modular static enforcement. In Proceedings of the IEEE Symposium on Security and Privacy. IEEE, Los Alamitos, CA.

Gilles Barthe, Salvador Cavadini, and Tamara Rezk. 2008. Tractable enforcement of declassification policies. In Proceedings of the 21st IEEE Computer Security Foundations Symposium. IEEE, Los Alamitos, CA.

David Basin, Jürgen Doser, and Torsten Lodderstedt. 2006. Model driven security: From UML models to access control infrastructures. ACM Transactions on Software Engineering and Methodology 15, 1, 3991.

David Elliott Bell and Leonard J. LaPadula. 1976. Secure Computer System: Unified Exposition and Multics Interpretation. Technical Report ESD-TR-75-306. Mitre Corporation, Bedford, MA.

David Bibighaus. 2006. Applying the Doubly Labeled Transition System to the Refinement Paradox. Ph.D. Dissertation. Naval Postgraduate School, Monterey, CA.

Holger Blasum, Sergey Tverdyshev, Bruno Langenstein, Jonas Maebe, Bjorn De Sutter, Bertrand Leconte, Benoit Triquet, Kevin Mller, Michael Paulitsch, Axel Sding-Freiherr von Blomberg, and Axel Tillequin. 2014. EUROMILS: MILS Architecture White Paper. Available at http://www.euromils.eu.

Carolyn Boettcher, Raytheon DeLong, John Rushby, and Wilmar Sifre. 2008. The MILS component integration approach to secure information sharing. In Proceedings of the 27th IEEE/AIAA Digital Avionics Systems Conference. 1.C.2-1-1.C.2-14.

Annalisa Bossi, Ricardo Focardi, Carlo Piazza, and Sabina Rossi. 2003. Refinement operators and information flow security. In Proceedings of the International Conference on Software Engineering and Formal Methods. 44-53.

Denis Bytschkow, Jean Quilbeuf, Georgeta Igna, and Harald Ruess. 2014. Distributed MILS architectural approach for secure smart grids. In Proceedings of the 2nd International Workshop on Smart Grid Security. 16-29.

Alexander G. Camek, Christian Buckl, and Alois Knoll. 2013. Future cars: Necessity for an adaptive and distributed multiple independent levels of security architecture. In Proceedings of the 2nd ACM International Conference on High Confidence Networked Systems. 17-24.

Stephen Chong and Ron van der Meyden. 2009. Deriving epistemic conclusions from agent architecture. In Proceedings of the Conference on Theoretical Aspects of Rationality and Knowledge (TARK'09). 61-70. 
Ronald Fagin, Joseph Y. Halpern, Yoram Moses, and Moshe Y. Vardi. 1995. Reasoning about Knowledge. MIT Press, Cambridge, MA.

Riccardo Focardi and Roberto Gorrieri. 1994. A classification of security properties for process algebras. Journal of Computer Security 3, 1, 5-33.

David Garlan, Robert T. Monroe, and David Wile. 2000. Acme: Architectural description of componentbased systems. In Foundations of Component-Based Systems, G. T. Leavens and M. Sitaraman (Eds.). Cambridge University Press, 47-68.

Joseph A. Goguen and Jose Meseguer. 1982. Security policies and security models. In Proceedings of the IEEE Symposium on Security and Privacy. IEEE, Los Alamitos, CA, 11-20.

Joseph A. Goguen and Jose Meseguer. 1984. Unwinding and inference control. In Proceedings of the IEEE Symposium on Security and Privacy. IEEE, Los Alamitos, CA, 75-86.

John Graham-Cumming and Jeff Sanders. 1991. On the refinement of noninterference. In Proceedings of the IEEE Computer Security Foundations Workshop. 35-42.

David Greve, Matthew Wilding, and W. Mark Vanfleet. 2003. A separation kernel formal security policy. In Proceedings of the 4th International Workshop on the ACL2 Prover and Its Applications.

Joshua D. Guttman and Paul D. Rowe. 2014. A cut principle for information flow. arXiv:1410.4617.

J. Thomas Haigh and William D. Young. 1987. Extending the noninterference version of MLS for SAT. IEEE Transactions on Software Engineering 13, 2, 141-150.

Jorgen Hansson, Peter H. Feiler, and John Morley. 2008. Building secure systems using model-based engineering and architectural models. CrossTalk: The Journal of Defense Software Engineering 21, 9, 12.

Constance L. Heitmeyer, Myla Archer, Elizabeth I. Leonard, and John McLean. 2006. Formal specification and verification of data separation in a separation kernel for an embedded system. In Proceedings of the 13th ACM Conference on Computer and Communications Security. ACM, New York, NY, 346-355.

Thomas H. Hinke and Marvin Schaefer. 1975. Secure Data Management System. Technical Report RADCTR-75-266. System Development Corporation.

Galen C. Hunt and James R. Larus. 2007. Singularity: Rethinking the software stack. Operating Systems Review 41, 2, 37-49.

Jeremy Jacob. 1989. On the derivation of secure components. In Proceedings of the IEEE Symposium on Security and Privacy. 242-247.

Jan Jürjens. 2005. Secure Systems Development with UML. Springer.

Wolfgang Kampichler and Dieter Eier. 2014. A D-MILS console subsystem for advanced ATM communication services. In Proceedings of the IEEE/AIAA 33rd Digital Avionics Systems Conference (DASC'14). 6D2: $1-8$.

Gregor Kiczales. 1996. Aspect-oriented programming. ACM Computing Surveys 28, 4es, Article No. 154.

Alexander Lux and Heiko Mantel. 2009. Who can declassify? In Formal Aspects in Security and Trust. Lecture Notes in Computer Science, Vol. 5491. Springer, 35-49.

Heiko Mantel. 2001. Preserving information flow properties under refinement. In Proceedings of the IEEE Symposium on Security and Privacy. 78-91.

Heiko Mantel and Alexander Reinhard. 2007. Controlling the what and where of declassification in languagebased security. In Programming Languages and Systems. Lecture Notes in Computer Science, Vol. 4421. Springer, 141-156.

Daryl McCullough. 1990. A hookup theorem for multilevel security. IEEE Transactions on Software Engineering 16, 6, 563-568.

John McLean. 1996. A general theory of composition for a class of "possibilistic" properties. IEEE Transactions on Software Engineering 22, 1, 53-67.

Carroll Morgan. 2006. The shadow knows: Refinement of ignorance in sequential programs. In Mathematics of Program Construction. Lecture Notes in Computer Science, Vol. 4014. Springer, 359-378.

Mark Moriconi and Xialei Qian. 1994. Correctness and composition of software architectures. In Proceedings of the 2nd ACM SIGSOFT Symposium on Foundations of Software Engineering. 164-174.

Mark Moriconi, Xialei Qian, and Robert A. Riemenschneider. 1995. Correct architecture refinement. IEEE Transactions on Software Engineering 21, 4, 356-372.

Mark Moriconi, Xialei Qian, Robert A. Riemenschneider, and Li Gong. 1997. Secure software architectures. In Proceedings of the IEEE Symposium on Security and Privacy. 84-893.

Kevin Mueller, Michael Paulitsch, Sergey Tverdyshev, and Holger Blasum. 2012. MILS-related information flow control in the avionic domain: A view on security-enhancing software architectures. In Proceedings of the IEEE/IFIP International Conference on Dependable Systems and Networks Workshops. 1-6. 
Toby C. Murray, Daniel Matichuk, Matthew Brassil, Peter Gammie, Timothy Bourke, Sean Seefried, Corey Lewis, Xin Gao, and Gerwin Klein. 2013. seL4: From general purpose to a proof of information flow enforcement. In Proceedings of the IEEE Symposium on Security and Privacy. 415-429.

Colin O'Halloran. 1992. Refinement and confidentiality. In Proceedings of the 5th Refinement Workshop. 119-139.

Kevin R. O’Neill. 2006. Security and Anonymity in Interactive Systems. Ph.D. Dissertation. Cornell University, Ithaca, NY.

Andrew W. Roscoe. 1995. CSP and determinism in security modelling. In Proceedings of the IEEE Symposium on Security and Privacy. 114-221.

Andrew W. Roscoe and Michael H. Goldsmith. 1999. What is intransitive noninterference? In Proceedings of the 12th IEEE Computer Security Foundations Workshop. IEEE, Los Alamitos, CA.

John Rushby. 1992. Noninterference, Transitivity, and Channel-Control Security Policies. Technical Report CSL-92-02. SRI International.

Andrei Sabelfeld and Andrew Myers. 2003. Language-based information-flow security. IEEE Journal on Selected Areas in Communications 21, 1, 5-19.

Andrei Sabelfeld and David Sands. 2005. Dimensions and principles of declassification. In Proceedings of the 18th IEEE Computer Security Foundations Workshop. IEEE, Los Alamitos, CA, 255-269.

Thomas Santen. 2008. Preservation of probabilistic information flow under refinement. Information and Computation 206, 2-4, 213-249.

Fredrik Seehusen and Ketil Stolen. 2006. Information flow property preserving transformation of UML interaction diagrams. In Proceedings of the ACM Symposium on Access Control Models and Technologies. 150-159.

Bhavani M. Thuraisingham. 2005. Database and Applications Security: Integrating Information Security and Data Management. CRC Press.

Ron van der Meyden. 2007. What, indeed, is intransitive noninterference? In Computer Security-ESORICS 2007. Lecture Notes in Computer Science, Vol. 4734. Springer, 235-250.

Ron van der Meyden. 2012. Architectural refinement and notions of intransitive noninterference. Formal Aspects of Computing 24, 4-6, 769-792.

W. Mark Vanfleet, R. William Beckworth, Ben Calloni, Jahn A. Luke, Carol Taylor, and Gordon Uchenick. 2005. MILS: Architecture for high assurance embedded computing. CrossTalk: The Journal of Defense Software Engineering 18, 12-16.

Armin Wasicek and Thomas Mair. 2013. Secure information sharing in mixed criticality systems. In Proceedings of the IAENG World Conference on Engineering and Science.

Jie Zhou and Jim Alves-Foss. 2006. Architecture-based refinements for secure computer system design. In Proceedings of the International Conference on Privacy, Security, and Trust: Bridge the Gap between PST Technologies and Business Services. Article No. 15.

Received October 2014; revised September 2015; accepted September 2015 